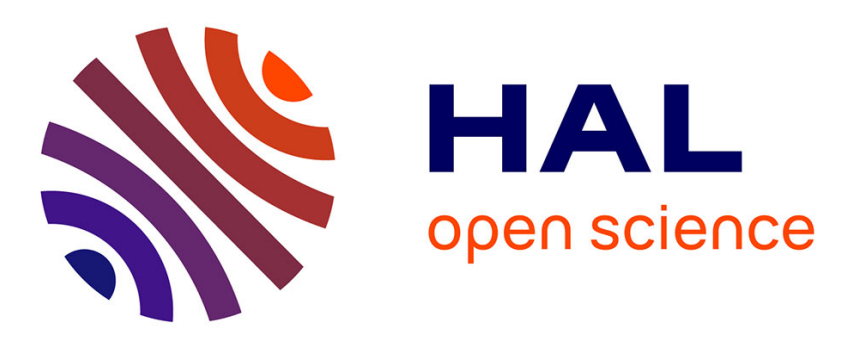

\title{
Electron exchange energy of neutral donors inside a quantum well
}

G. Garcia-Arellano, Frédéric Bernardot, C. Testelin, M. Chamarro

\section{To cite this version:}

G. Garcia-Arellano, Frédéric Bernardot, C. Testelin, M. Chamarro. Electron exchange energy of neutral donors inside a quantum well. Physical Review B: Condensed Matter and Materials Physics (1998-2015), 2018, 98 (19), 10.1103/PhysRevB.98.195308 . hal-02390029

\section{HAL Id: hal-02390029 \\ https://hal.science/hal-02390029}

Submitted on 14 Jul 2020

HAL is a multi-disciplinary open access archive for the deposit and dissemination of scientific research documents, whether they are published or not. The documents may come from teaching and research institutions in France or abroad, or from public or private research centers.
L'archive ouverte pluridisciplinaire HAL, est destinée au dépôt et à la diffusion de documents scientifiques de niveau recherche, publiés ou non, émanant des établissements d'enseignement et de recherche français ou étrangers, des laboratoires publics ou privés. 


\title{
Electron exchange energy of neutral donors inside a quantum well
}

\author{
G. Garcia-Arellano, F. Bernardot, C. Testelin, and M. Chamarro \\ Sorbonne Université, CNRS, Institut des NanoSciences de Paris, 4 place Jussieu, F-75005 Paris, France
}

(Received 24 July 2018; revised manuscript received 15 October 2018; published 19 November 2018)

\begin{abstract}
We calculated the exchange energy of a pair of donor-bound electrons placed in the middle of an infinite quantum well (QW). In order to obtain this energy for any interdonor distance and for any QW thickness, we have first adapted to a QW the method developed by Gor'kov and Pitaevskii [L. P. Gor'kov and L. P. Pitaevskii, Dokl. Akad. Nauk SSSR 151, 822 (1963)] for a three-dimensional (3D) distribution of donors, and calculated the asymptotic form of the exchange energy. Second we have calculated the exchange energy of a "helium atom" in a QW; and third, inspired by the interpolation procedure proposed by Ponomarev et al. [I.V. Ponomarev et al., Phys. Rev. B 60, 5485 (1999)], we have obtained an interpolated expression for any interdonor distance. The obtained exchange energy is written in units of effective hartree, and the distance between the donors, as well as the width of the QW, are expressed in units of effective Bohr radius. We calculated the exchange energy for some commonly studied semiconductor materials, and discussed also the relationship between the exchange energy and the spin relaxation time for a donor concentration close to the insulator-metal transition.
\end{abstract}

DOI: 10.1103/PhysRevB.98.195308

\section{INTRODUCTION}

The association of the carrier-carrier Coulomb interaction and the antisymmetric character of the state of an electron pair are at the foundation of the exchange interaction between localized electrons; this interaction is one of the oldest topics in quantum mechanics. In the last decade, the strong interest in controlling and manipulating localized spins in solid-state systems, such as quantum dots or impurities, has renewed this topic. Indeed, since the birth of the idea of a quantum computer $[1,2]$ numerous efforts, both theoretical and experimental, have been made to physically identify qubit candidates. Solid-state qubits are a subset of qubits which present the advantage of scalability due to the use of nanofabrication technologies, but which also present challenges to obtain a suitable protection against interactions from their environment. Another issue is to fabricate qubits identical to each other, and to make them properly communicate. Nowadays, the two main qubit technologies concern superconductor and semiconductor materials, for which several qubits have been identified. In superconducting circuits obtained with nanotechnologies similar to those of the microelectronics, qubits based on phase, charge of flux states have reached a spectacular degree of quantum control [3,4]. One of the main requirements for qubits is to show long coherence times, and then in solid-state physics these qubits have to be protected against environmental interactions. In this sense, the spin of the low-energy electronic states in semiconductors is, in principle, an observable well protected from the environment, and constitutes a good prototype of a qubit. Moreover, to suppress the relaxation mechanism of itinerant electrons (D'yakonov-Perel process), the electron spin should be localized at a nanometer scale. The confinement can be obtained by nanofabrication as in quantum dots [5-8], or in a more natural way using the attractive potential of individual impurities [9-13]. Some years ago, electrons trapped by individual donors in semiconductors at low temperature emerged as one of the possible candidates [8,14-17]. In particular, it has been experimentally demonstrated that, when donors are immersed in a quantum well $(\mathrm{QW})$, the spin relaxation time of electrons localized on donors enhances by two orders of magnitude with respect to spin relaxation time of free electrons in QWs [18]. Moreover, by inserting the donors in a QW, the optical selection rules for circularly polarized light are purified, allowing a higher degree of optical orientation of the electron spins than in 3D crystals [18-21].

In this framework, two main challenges are related with the exchange energy between spin qubits: (i) the enhancement of the relaxation and coherence times, and (ii) the mechanism of the entanglement in a pair of qubits. The spin relaxation time is strongly related to the distance between two electrons localized on donors. Several experimental and theoretical studies have addressed this issue [12,22-24]. Different mechanisms contribute to the spin relaxation: at very low concentration of donors, the coupling of an isolated electron spin with the surrounding nuclear spins is the dominant interaction, and at donor concentrations near the metallic transition the exchange interaction is the main mechanism of the spin-spin interaction between neighboring donors. Controlled entanglement requires a well-known interaction between spin qubits, the strength of which fixes the speed of a two-qubit gate. Different mechanisms of entanglement have been proposed, as for example coupling to photons via a cavity mode [25,26], coupling to virtual excitations of delocalized exciton states [27], dipoledipole interaction between charged excitons strongly polarized by an external dc field [28], or the exchange interaction between two electrons in neighboring qubits $[8,9,29]$.

The most appropriate method to get the exchange energy in the limit of large distances between donors was developed by Gor'kov and Pitaevskkii, and Herring and Flicker [30,31]. In this method, the authors calculated the exchange energy between two "hydrogen atoms", reducing its expression to a hyperplane integral in a six-dimensional space, and finally 
getting an analytical formula in 3D. Later, Ponomarev et al. [32] proposed a procedure to obtain an expression valid for any interdonor distance. This procedure is based on an interpolation between the exchange energy at zero distance (i.e., the "helium atom") and the known asymptotic behavior. The same authors also calculated the asymptotic and interpolated exchange energies for artificial two dimensional (2D) systems: electrons confined to the same plane as the impurities, and electrons bound to Coulomb centers which are located outside the plane [33].

In this paper, we have calculated the exchange energy of a pair of donor-bound electrons placed in the middle of an infinite QW, for any interdonor distance and for different QW thicknesses. We calculated first, in Sec. II, the asymptotic form of the exchange energy, adapting to a QW the method developed in Refs. [30,31]. In Sec. III, inspired by the interpolation procedure proposed by Ponomarev et al. [32], we calculated the exchange energy of a "helium atom" inside an infinite QW, and finally reached the values of the exchange energy valid for any interdonor distance and for any QW thickness. In Sec. IV, we discussed the behavior of the exchange energy in different III-V and II-VI materials, and its relationship with the spin relaxation time at donor concentrations close to the insulator-metal transition.

\section{EXCHANGE ENERGY FOR LARGE DISTANCES BETWEEN DONORS}

\section{A. General framework}

We consider two electrons, 1 and 2, localized at low temperature on two donors $A$ and $B$. Both donors are located at fixed positions $x_{A}=-a$ and $x_{B}=+a$ in a semiconductor matrix. The Hamiltonian describing the system of both donorbound electrons is

$$
\widehat{H}=-\frac{\Delta_{1}}{2}-\frac{\Delta_{2}}{2}-\frac{1}{r_{1 A}}-\frac{1}{r_{2 B}}-\frac{1}{r_{1 B}}-\frac{1}{r_{2 A}}+\frac{1}{r_{12}}+\frac{1}{2 a},
$$

where $\Delta_{j}$ is the Laplacian operator acting on electron $j$ ( $j=$ 1 or 2), $r_{j A}$ and $r_{j B}$ are the distances of electron $j$ to donors $A$ and $B$, respectively, and $r_{12}=\left|\vec{r}_{2}-\vec{r}_{1}\right|$ is the distance between the electrons. In the following, the Cartesian coordinates $x_{j}, y_{j}, z_{j}$ of electron $j(j=1$ or 2 ) will be employed. Here, and in the following, the distances are expressed in units of effective (bulk) Bohr radius $a_{B}^{*}$, and the energies in units of effective hartree $E_{h}{ }^{*} ; a_{B}{ }^{*}$ and $E_{h}{ }^{*}$ are defined as follows: $a_{B}^{*}=a_{0} \varepsilon_{r}\left(m / m^{*}\right)$, with $a_{0}=4 \pi \varepsilon_{0} \hbar^{2} / m e^{2} \approx 0.52918 \AA$ the Bohr radius of the atomic units, and $E_{h}{ }^{*}=E_{h}\left(m^{*} / m \varepsilon_{r}^{2}\right)$, where $E_{h}=e^{2} / 4 \pi \varepsilon_{0} a_{0} \approx 27.211 \mathrm{eV}$ is the hartree energy of the atomic units; $m$ is the electron mass, $m^{*}$ the effective electron mass, and $\varepsilon_{r}$ the dielectric constant.

The Hamiltonian $\hat{H}$ being independent on the electron spins $\vec{S}_{1}$ and $\vec{S}_{2}$, the Pauli principle implies that the twoelectron states are singlet states $\Psi=\Psi_{S}\left|S=0, S_{z}=0\right\rangle$, with an orbital part $\Psi_{S}$ symmetric by electron exchange $\left[\Psi_{S}\left(\vec{r}_{2}, \vec{r}_{1}\right)=+\Psi_{S}\left(\vec{r}_{1}, \vec{r}_{2}\right)\right]$, or triplet states $\Psi=$ $\Psi_{A}\left|S=1, S_{z}=0, \pm 1\right\rangle$, with an orbital part $\Psi_{A}$ antisymmetric by electron exchange $\left[\Psi_{A}\left(\vec{r}_{2}, \vec{r}_{1}\right)=-\Psi_{A}\left(\vec{r}_{1}, \vec{r}_{2}\right)\right] ; \vec{S}=$ $\vec{S}_{1}+\vec{S}_{2}$ is the total spin of the pair of electrons. As it is well know that the ground state of such a system is a singlet and the first-excited one a triplet, the notations $\Psi_{S}$ and $E_{S}$ designate from now on the wavefunction and energy of the ground state, and $\Psi_{A}$ and $E_{A}$ the ones of the first-excited state.

The energy difference between the first-excited and the ground levels, $E_{A}-E_{S}=2 \mathrm{~J}$, is called the exchange energy, or exchange splitting, of the two-electron system. We are using here, and in all the following, a positively defined exchange energy, $2 J>0$ : the Hamiltonian $\hat{H}$ of Eq. (1) then corresponds to the spin Hamiltonian $\hat{H}_{\text {exc }}=+2 J \vec{S}_{1} \cdot \vec{S}_{2}$. Let us stress that the spin-orbit interaction, not taken into account in Eq. (1), brings additional anisotropic contributions in $\hat{H}_{\text {exc }}$, see Refs. [24,34]. We do not write them here, because we are concerned by the isotropic part of $\hat{H}_{\text {exc }}$; they are considered in Sec. IV.

The calculation of the exchange energy $2 J$, in the limit of large distances $R=2 a$ between donors, was first explored in the 1920's [35]. A more refined, and more correct, method was later developed in Refs. [30,31] for doped bulk materials (3D case), and also used in the purely 2D case (see Refs. [32,33]) of two donors placed in a plane. This latter method yields an integral expression of the exchange energy:

$$
2 J(R \gg 1)=\int_{\Sigma} \Psi_{2} \vec{\nabla} \Psi_{1} \cdot d \Sigma,
$$

with $\Psi_{1}=\Psi_{S}+\Psi_{A}$ and $\Psi_{2}\left(\vec{r}_{1}, \vec{r}_{2}\right)=\Psi_{1}\left(\vec{r}_{2}, \vec{r}_{1}\right)$, the integral being on the hyperplane $\Sigma$ defined by $x_{2}=x_{1}$, and the elemental hypersurface $d \vec{\Sigma}$ pointing towards the $x_{2}>x_{1}$ region. At this stage, $\Psi_{1}$ can be approximated in Eq. (2) by the expression

$$
\Psi_{1}\left(\vec{r}_{1}, \vec{r}_{2}\right)=\phi_{A}\left(\vec{r}_{1}\right) \phi_{B}\left(\vec{r}_{2}\right) \chi\left(\vec{r}_{1}, \vec{r}_{2}\right),
$$

where $\phi_{A}(\vec{r})=\phi_{1}\left(\vec{r}-\vec{R}_{A}\right)$ is the one-electron ground-state wavefunction on donor $A$, located at $\vec{R}_{A}, \phi_{B}(\vec{r})=\phi_{1}\left(\vec{r}-\vec{R}_{B}\right)$ is the one for donor $B$, located at $\vec{R}_{B}$, and $\chi\left(\vec{r}_{1}, \vec{r}_{2}\right)$ is a slowly varying correlation function manifesting that the two electrons are avoiding each other: $\chi\left(\vec{r}_{1}, \vec{r}_{2}\right)=0$ for $\vec{r}_{1}=\vec{r}_{2}$. The $\chi$ function is first determined, and so the calculations in $3 \mathrm{D}$ and $2 \mathrm{D}$ have been achieved by inserting expression (3) in Eq. (2), see Eqs. (10).

\section{B. Calculation in an infinite quantum well}

The case of two donors placed in the mid-plane $z=0$ of an infinite QW, of thickness $L$, introduces an extrinsic confinement of the 3D one-electron wavefunctions. This situation is intermediate between those at $3 \mathrm{D}$ and $2 \mathrm{D}$, with $L$ as a variable parameter. Here, we employed the one-electron ground-state wavefunction $\phi_{1}(\vec{r})$ studied in Appendix A, with atomic number $Z=1$. Then, following the steps of the method developed to reach $2 J(R \gg 1)$ in 3D (Ref. [30]), it turns out that the function $\chi\left(\vec{r}_{1}, \vec{r}_{2}\right)$ obeys the equation

$$
\alpha_{1}\left(\frac{\partial \chi}{\partial x_{1}}-\frac{\partial \chi}{\partial x_{2}}\right)+\left(\frac{1}{2 a}+\frac{1}{r_{12}}-\frac{1}{a-x_{1}}-\frac{1}{a+x_{2}}\right) \chi=0
$$

considering the positions of both electrons near the $x$ axis, with $x_{1}>-a$ and $x_{2}<a ; \alpha_{1}$ is the radial decreasing rate of $\phi_{1}\left(\right.$ see Appendix A) - in 3D, $\alpha_{1}=1-$. So it appears that 
the function $\chi^{\alpha_{1}}$ obeys the equation met in 3D. Considering the limit conditions $\chi \rightarrow 1$ when $x_{1} \rightarrow-a$ or $x_{2} \rightarrow a$, this means that $\chi^{\alpha_{1}}$ coincides with the 3D correlation function $\chi_{3 \mathrm{D}}$. Using the known expression of $\chi_{3 \mathrm{D}}\left(\vec{r}_{1}, \vec{r}_{2}\right)$, see Ref. [30] or Appendix D, we hence deduced

$$
\chi\left(\vec{r}_{1}, \vec{r}_{2}\right)=\chi_{3 \mathrm{D}}\left(\vec{r}_{1}, \vec{r}_{2}\right)^{1 / \alpha_{1}} .
$$

Noticeably, this expression of $\chi\left(\vec{r}_{1}, \vec{r}_{2}\right)$ goes to the right $3 \mathrm{D}$ limit [30] when $\alpha_{1} \rightarrow 1$ (for $L \rightarrow \infty$ ), and also to the right 2D limit [32,33] when $\alpha_{1} \rightarrow 2$ (for $L \rightarrow 0$ ).

We are now able to express the exchange energy $2 J(R \gg 1)$ in its integral form (2). Retaining only derivatives of $\phi_{1}$ with respect to $x_{1}$ and $x_{2}$, and neglecting the terms containing the derivatives of $\chi$, we are first conducted to

$$
\begin{aligned}
2 J= & 8 \alpha_{1} \int_{0}^{a} d x_{1} \int_{-\infty}^{+\infty} d y_{1} d y_{2} \\
& \times\left.\left.\int_{\left|z_{1,2}\right|<L / 2} d z_{1} d z_{2} \Psi_{1}\left(\vec{r}_{2}, \vec{r}_{1}\right)\right|_{x_{2}=x_{1}} \Psi_{1}\left(\vec{r}_{1}, \vec{r}_{2}\right)\right|_{x_{2}=x_{1}},
\end{aligned}
$$

and then to

$$
\begin{aligned}
2 J(R \gg 1)= & 4 \alpha_{1}\left(\frac{8}{\mathrm{e}}\right)^{\frac{1}{\alpha_{1}}} A_{1}^{4} R^{3-\frac{1}{2 \alpha_{1}}} \mathrm{e}^{-2 \alpha_{1} R} \int_{0}^{1} d X \\
& \times \int_{\left|Z_{1,2}\right|<\frac{L}{2 \sqrt{R}}} d Z_{1} d Z_{2} \int_{-\infty}^{+\infty} d Y_{1} d Y_{2}
\end{aligned}
$$

$$
\begin{aligned}
& \times \cos ^{2}\left(\frac{\pi \sqrt{R} Z_{1}}{L}\right) \cos ^{2}\left(\frac{\pi \sqrt{R} Z_{2}}{L}\right) \\
& \times \exp \left\{-\frac{2 \alpha_{1}\left(P_{1}^{2}+P_{2}^{2}\right)}{1-X^{2}}+\frac{X}{\alpha_{1}}\right\} \\
& \times\left[\frac{P_{12}}{(1+X)^{2}(1-X)}\right]^{\frac{1}{\alpha_{1}}},
\end{aligned}
$$

using $R=2 a$, the change of variables $x_{1}=a X, y_{1,2}=$ $Y_{1,2} \sqrt{R}, z_{1,2}=Z_{1,2} \sqrt{R}$, and the notations $P_{j}=\sqrt{Y_{j}^{2}+Z_{j}^{2}}$ $(j=1$ or 2$)$ and $P_{12}=\sqrt{\left(Y_{1}-Y_{2}\right)^{2}+\left(Z_{1}-Z_{2}\right)^{2}}$.

The expression (7) of the exchange energy $2 J(R \gg 1)$ can be calculated numerically, for given values of the thickness $L$ of the QW (for a given $L, \alpha_{1}$ and $A_{1}$ are known, see Appendix A). In order to decrease the numerical integration time, we turned the 5-dimensional integral (7) to a 4-dimensional one, using the change of variables

$$
\begin{aligned}
S_{1}=\frac{Y_{1}+Y_{2}}{2}, & S_{2}=\frac{Z_{1}+Z_{2}}{2}, \\
D_{1}=Y_{1}-Y_{2}, & D_{2}=Z_{1}-Z_{2} ;
\end{aligned}
$$

the integration over variable $S_{1}$ can be performed, and we finally used the following expression (9) for our numerical calculations:

$$
\begin{aligned}
2 J(R \gg 1)= & 4 \sqrt{\pi \alpha_{1}}\left(\frac{8}{\mathrm{e}}\right)^{\frac{1}{\alpha_{1}}} A_{1}^{4} R^{3-\frac{1}{2 \alpha_{1}}} \mathrm{e}^{-2 \alpha_{1} R} \int_{0}^{1} d X \sqrt{1-X^{2}}\left[\frac{\mathrm{e}^{X}}{(1+X)^{2}(1-X)}\right]^{\frac{1}{\alpha_{1}}} \\
& \times \int_{0}^{+\infty} d D_{1} \int_{0}^{\frac{L}{\sqrt{R}}} d D_{2}\left(D_{1}^{2}+D_{2}^{2}\right)^{\frac{1}{2 \alpha_{1}}} \exp \left[-\frac{\alpha_{1}\left(D_{1}^{2}+D_{2}^{2}\right)}{1-X^{2}}\right] \\
& \times\left\{\cos ^{2}\left(\frac{\pi \sqrt{R} D_{2}}{L}\right) \int_{0}^{\frac{L}{2 \sqrt{R}}-\frac{D_{2}}{2}} d S_{2} \exp \left[-\frac{4 \alpha_{1} S_{2}^{2}}{1-X^{2}}\right]\right. \\
& +2 \cos \left(\frac{\pi \sqrt{R} D_{2}}{L}\right) \int_{0}^{\frac{L}{2 \sqrt{R}}-\frac{D_{2}}{2}} d S_{2} \cos \left(\frac{2 \pi \sqrt{R} S_{2}}{L}\right) \exp \left[-\frac{4 \alpha_{1} S_{2}^{2}}{1-X^{2}}\right] \\
& \left.+\int_{0}^{\frac{L}{2 \sqrt{R}}-\frac{D_{2}}{2}} d S_{2} \cos ^{2}\left(\frac{2 \pi \sqrt{R} S_{2}}{L}\right) \exp \left[-\frac{4 \alpha_{1} S_{2}^{2}}{1-X^{2}}\right]\right\}
\end{aligned}
$$

It is easy to check that, when $L \rightarrow \infty$ or $L \rightarrow 0$, Eq. (9) goes to the formulas known in $3 \mathrm{D}$ or $2 \mathrm{D}$, respectively (see Refs. [31-33]):

$$
\begin{aligned}
& 2 J_{3 \mathrm{D}}(R \gg 1)=1.6366 R^{5 / 2} \mathrm{e}^{-2 R} ; \\
& 2 J_{2 \mathrm{D}}(R \gg 1)=30.413 R^{7 / 4} \mathrm{e}^{-4 R} .
\end{aligned}
$$

Figure 1 shows the exchange energy $2 J(R \gg 1)$ as a function of the distance $R=2 a$ between the pair of donors, for several values of the QW thickness $L$. The $2 J$ values for $R \sim 1$, and below, have a priori no physical significance, because we fixed $R \gg 1$ from the start.

\section{DETERMINATION OF $2 J(R)$ FOR ANY $R$}

\section{A. Procedure to get an interpolated expression of $2 J(R)$}

In Ref. [32], a procedure is proposed to build the exchange energy $2 J(R)$ for any value of the distance $R$ between the donors, in the 3D and $2 \mathrm{D}$ cases. This procedure makes use of (i) the asymptotic form of the exchange energy

$$
2 J(R \gg 1)=C R^{\beta} \mathrm{e}^{-\omega R},
$$

followed at 3D and 2D, see Eqs. (10); (ii) the value of the exchange energy $2 J_{0}=2 J(R=0)$ of the $3 \mathrm{D}$ and $2 \mathrm{D}$ helium atom; and (iii) two analytical assumptions: $\ln [2 J(R \ll 1)]$ 


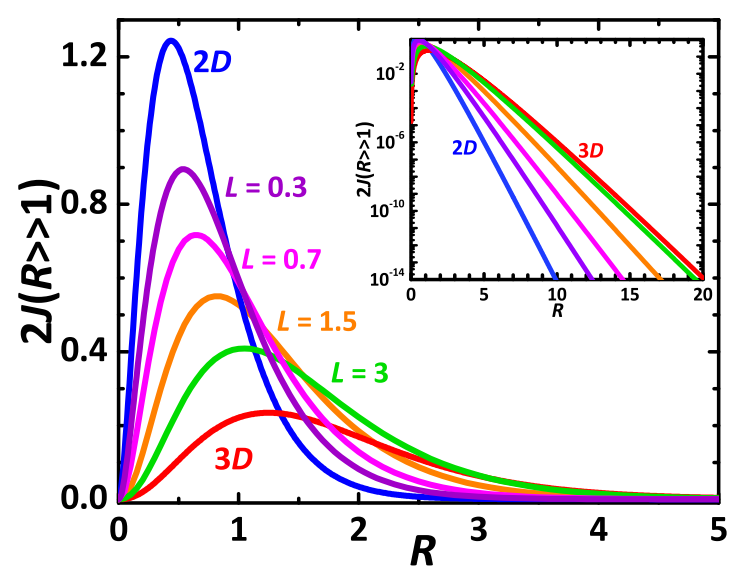

FIG. 1. Exchange energy $2 J(R \gg 1)$ for several values of the QW thickness $L$, as a function of the distance $R$ between two donorbound electrons, centered inside the QW. The curves noted 2D and 3D correspond to the $L \rightarrow 0$ and $L \rightarrow \infty$ limits, respectively. Inset: semilogarithmic representation of the same data, for a larger range of $R$.

can be expanded as $\ln \left(2 J_{0}\right)-\gamma R-\tilde{A} R^{2} \cdots$, with $\gamma$ and $\tilde{A}$ both positive, and the second derivative of $\ln [2 J(R)]$ is Lorentzian for any distance $R$ :

$$
\frac{d^{2} \ln [2 J(R)]}{d R^{2}}=-\frac{2 \tilde{A} \beta}{\beta+2 \tilde{A} R^{2}} .
$$

A first integration of Eq. (12) gives the expression of the first derivative of $\ln [2 J(R)]$ for any $R$, and a relationship between $\gamma$ and $\tilde{A}$ :

$$
A=\sqrt{2 \tilde{A} / \beta}=\frac{2(\omega-\gamma)}{\pi \beta} .
$$

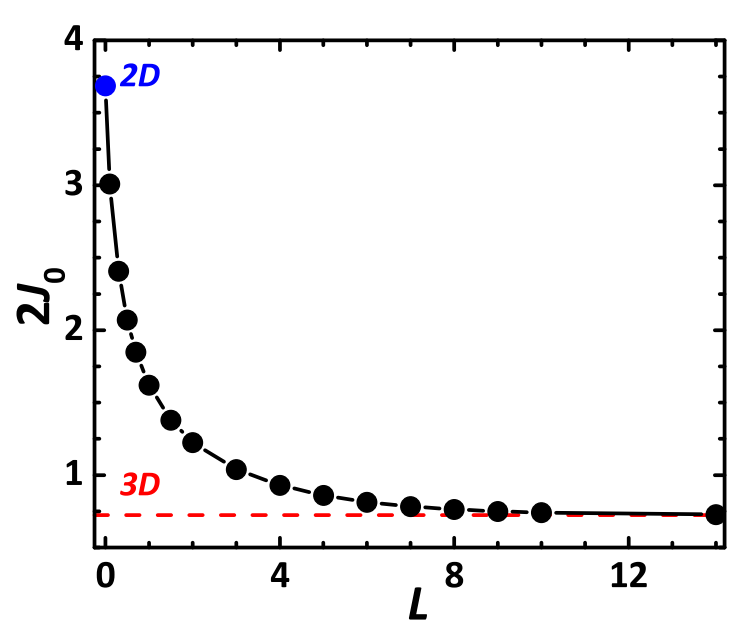

FIG. 2. Exchange energy $2 J_{0}$ of a "helium atom" (full disks) centered in an infinite QW, as a function of the QW thickness $L$. The blue point at $L=0$ corresponds to our calculated $2 J_{0}$ in $2 \mathrm{D}$; the red dashed horizontal line indicates the value of our calculated $2 J_{0}$ in 3D (continuous curve: guide for the eyes).

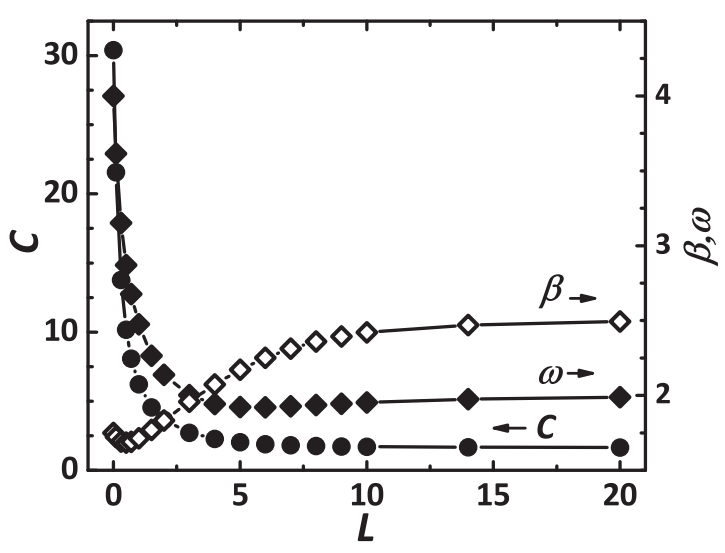

FIG. 3. Fitting parameters $C$ (full disks), $\beta$ (open diamonds), and $\omega$ (full diamonds) of the exchange energy $2 J(R \gg 1)$ with Eq. (11), as a function of the QW thickness $L$. The values of $C$ read on the left axis, the values of $\beta$ and $\omega$ on the right axis (continuous curves: guides for the eyes).

Then, a second integration yields the interpolated formula for $2 J(R)$ given by this procedure:

$$
\begin{aligned}
\ln [2 J(R)]= & \ln \left(2 J_{0}\right)-\gamma R-\beta A R \arctan (A R) \\
& +\frac{\beta}{2} \ln \left(1+A^{2} R^{2}\right) .
\end{aligned}
$$

One single parameter, $A$ (or $\gamma$ ), remains to be determined. In Ref. [32], the authors have chosen the parameter $A$ by fitting at best numerical values of $2 J$ obtained for $R$ of the order of unity or several units. So they proposed interpolated formulas for $2 J(R)$ in the $3 \mathrm{D}$ and $2 \mathrm{D}$ cases, using a $2 J_{0}$ value in $3 \mathrm{D}$ known at their time and, at $2 \mathrm{D}$, a value of $2 J_{0}$ calculated by their own. In Appendix C, we propose slightly modified expressions of $2 J(R)$ for the $3 \mathrm{D}$ and $2 \mathrm{D}$ cases.

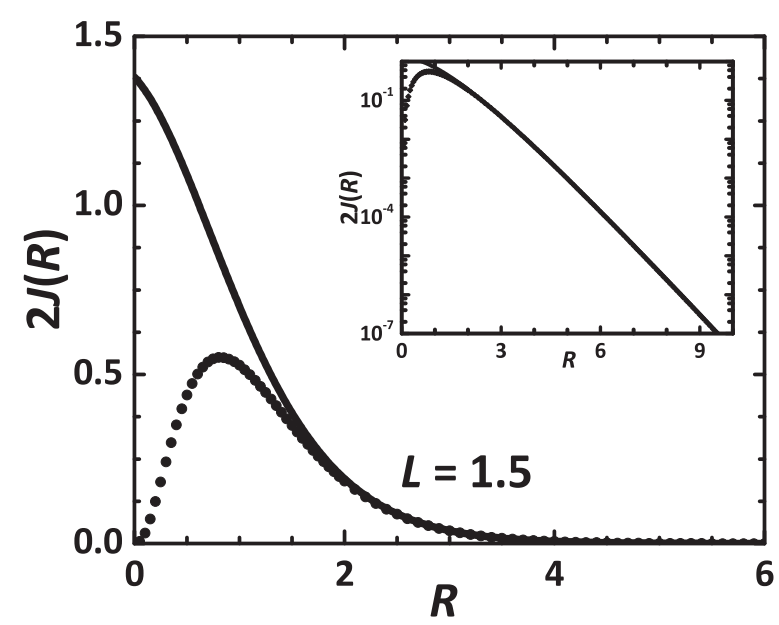

FIG. 4. Interpolated $2 J(R)$ (continuous curve) and large- $R$ $2 J(R \gg 1)$ (dotted curve) exchange energies for the QW thickness $L=1.5$, as a function of the distance $R$ between two donor-bound electrons, centered inside the QW. Inset: semilogarithmic representation of the same data, for a larger range of $R$. 


\section{B. Interpolated $2 J(R)$ in an infinite quantum well}

In the present study of the exchange energy between two donors located in the middle of an infinite QW, we faced two issues in order to build an interpolated $2 J(R)$ with the procedure described in Sec. III A: (i) the calculation of $2 J_{0}$ in a "helium atom" centred in the QW; (ii) the creation of the interpolated $2 J(R)$ starting with an asymptotic $2 J(R \gg 1)$, Eq. (9), which does not possess the standard form (11).

Let us first discuss the calculations we made to obtain $2 J_{0}$ in the middle of an infinite QW. We noticed that, in 3D and in $2 \mathrm{D}$, calculating the ground energy $E_{S}$ of the helium atom using the variational method, and the first-excited energy $E_{A}$ perturbatively, gives a very good approximation of the exchange energy $2 J_{0}$, which is found to be shifted from the exact numerical values by only about $1 \%$ (see Appendix B). So we proceeded in the same way to calculate the exchange energy of a "helium atom" located in the midplane of an infinite QW; our calculations of $E_{S}$ and $E_{A}$ are presented in Appendix B. Figure 2 shows $2 J_{0}$ as a function of the thickness $L$ of the QW; as it is observed, $2 J_{0}$ decreases monotonously with $L$, from the $2 \mathrm{D}$ value to the $3 \mathrm{D}$ one.

Let us now turn to the construction of the interpolated $2 J(R)$ in an infinite QW. The calculated asymptotic values of the exchange energy $2 J(R \gg 1)$, see Eq. (9) and Fig. 1, do not possess exactly the form (11) met in the 3D and $2 \mathrm{D}$ cases. But we realized that the fit of $2 J(R \gg 1)$ with Eq. (11) is rather satisfactory for values of $R$ ranging from $R=0.1$ to 30 ; the largest considered distance $R=30$ between the two donors corresponds to residual concentrations in typical semiconductors. Then we obtained the parameters $C, \beta$, and $\omega$ which are shown in Fig. 3; we reached them by adjusting $\ln [2 J(R \gg 1)]$ with $\ln C+\beta \ln R-\omega R$. Figure 3 shows these parameters as a function of the thickness of the QW. Finally, we performed a fit of the values of $2 J(R \gg 1)$ between $R=20$ and 30 with Eq. (14), using the known values of $2 J_{0}$ and $\beta ; A$ was the only fitting parameter $[\gamma$ is linked to $A$, once $\beta$ and $\omega$ are fixed, see Eq. (13)]. We created in this

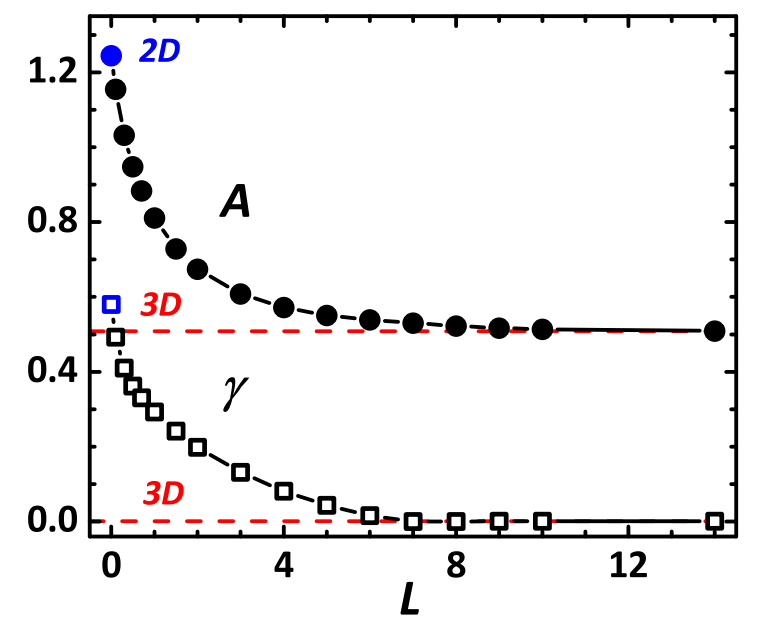

FIG. 5. Parameters $A$ (full disks) and $\gamma$ (open squares) entering in the expression of the interpolated $2 J(R)$ exchange energy, see Eq. (14), as a function of the QW thickness $L$ (continuous curves: guides for the eyes). The blue points at $L=0$ correspond to the 2D case; the red dashed horizontal lines indicate the values in $3 \mathrm{D}$.
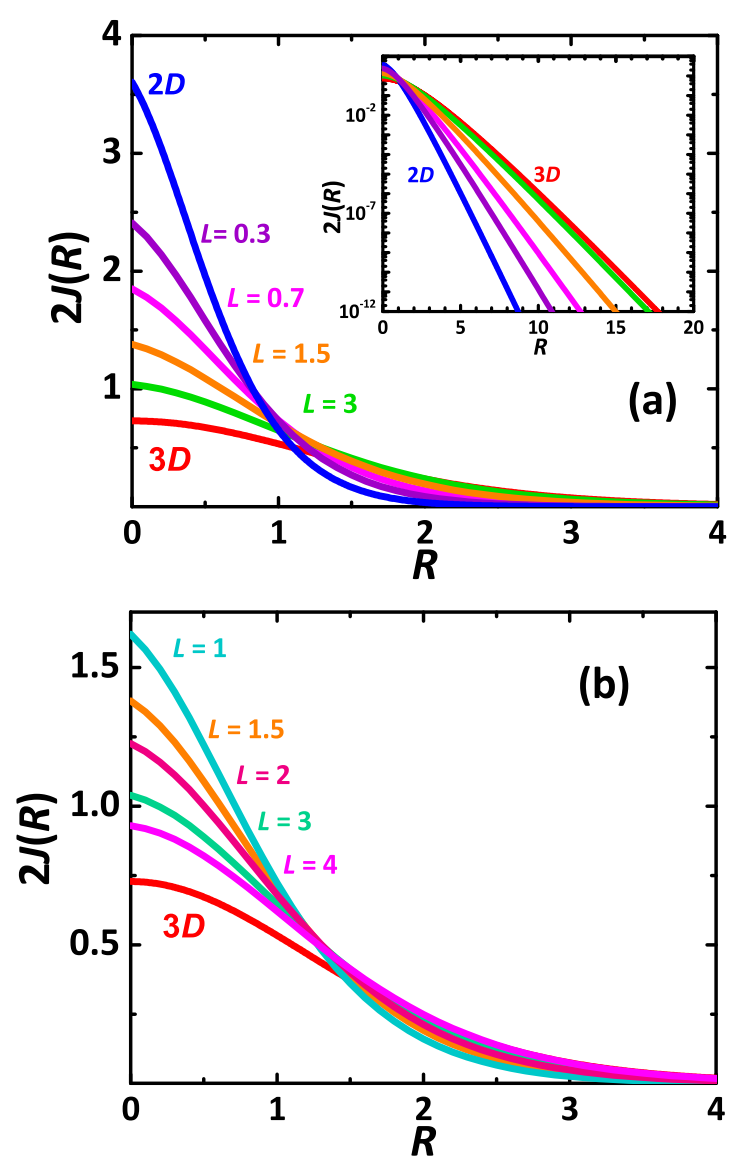

FIG. 6. (a) Interpolated exchange energy $2 J(R)$ for the $L$ values shown in Fig. 1, as a function of the distance $R$ between two donor-bound electrons, centered inside the QW. The curves noted $2 \mathrm{D}$ and $3 \mathrm{D}$ correspond to the interpolated $2 J(R)$ laws in $2 \mathrm{D}$ and in $3 \mathrm{D}$, respectively, see Eqs. (15). Inset: semilogarithmic representation of the same data, for a larger range of $R$. (b) Interpolated exchange energy $2 J(R)$ for $L=1,1.5,2,3,4$, and $3 \mathrm{D}$.

way an interpolated formula for $2 J(R)$ inside an infinite QW, for any distance $R$ of interest between the pair of donors. An example of interpolated exchange energy $2 J(R)$ is given in Fig. 4, for $L=1.5$; its coincidence with the asymptotic form $2 J(R \gg 1)$ occurs for $R \geqslant 2$.

As a special case, we recalculated the interpolated formulas in $3 \mathrm{D}$ and $2 \mathrm{D}$ following our procedure. We found

$$
\begin{aligned}
2 J_{3 \mathrm{D}}(R)= & 0.729\left(1+0.258 R^{2}\right)^{\frac{5}{4}} \exp [-0.0005 R \\
& -1.270 R \arctan (0.508 R)] ; \\
2 J_{2 \mathrm{D}}(R)= & 3.604\left(1+1.548 R^{2}\right)^{\frac{7}{8}} \exp [-0.579 R \\
& -2.178 R \arctan (1.244 R)] .
\end{aligned}
$$

These interpolated expressions are remarkably close to the ones calculated in Appendix C.

Figure 5 shows the values of the obtained parameters $A$ and $\gamma$, as a function of the QW thickness $L$; monotonous evolutions of $A$ and $\gamma$ can be observed between the 2D $(L \rightarrow 0)$ and 3D $(L \rightarrow \infty)$ cases. For $L \geqslant 6$, the $A$ and $\gamma$ values are very close to the values at 3D. In Fig. 6, we plotted the interpolated exchange energy $2 J(R)$ for several values of 

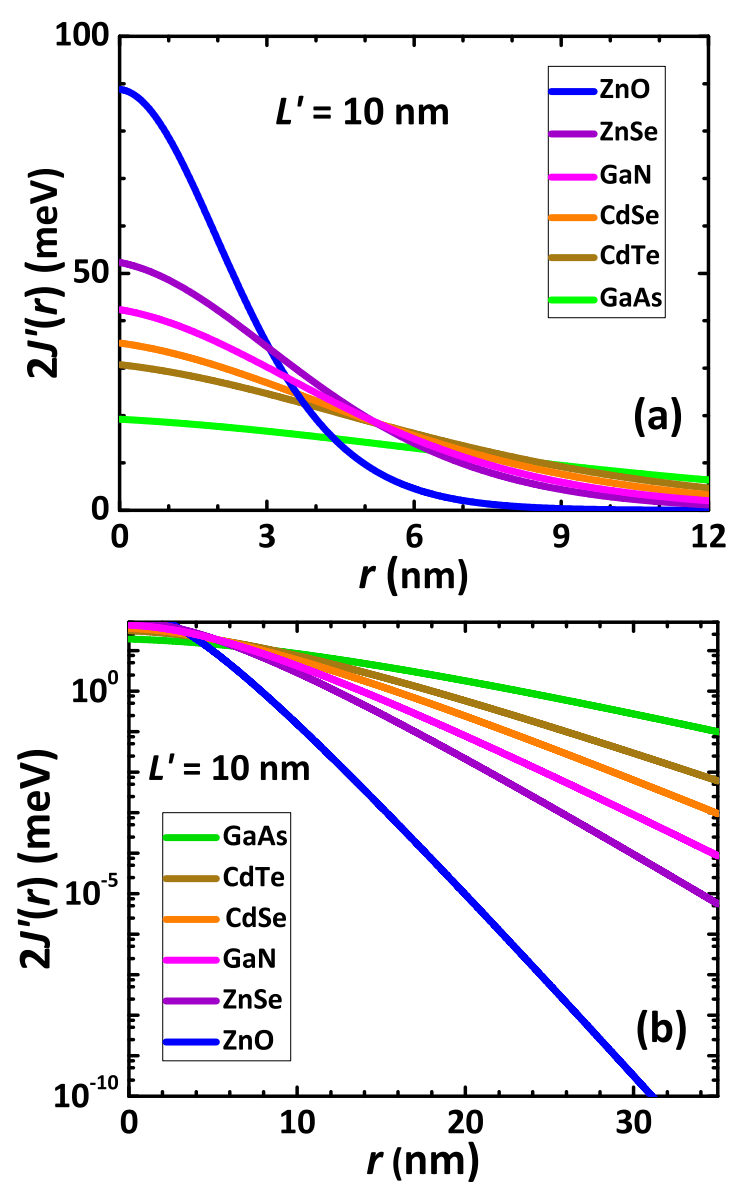

FIG. 7. Electron exchange energy in a common unit of energy, as a function of interdonor distance for several semiconductor materials, at a fixed value of QW thickness $L^{\prime}=10 \mathrm{~nm}$; (a) in linear scales and for small interdonor distances; (b) in semilogarithmic scales and for a larger domain of interdonor distances.

$L$, as a function of the distance $R$ between two donor-bound electrons, centered inside the QW. Because we consider an infinite QW with a wavefunction completely confined in the QW, our calculations do not describe correctly real systems for $L<1$. Indeed, the electron wavefunction of thin QWs, for which $L^{\prime}=L a_{B}^{*}$ is smaller than $a_{B}^{*}$, is in fact larger than the QW thickness, and then overflows the barrier material which does not represent an infinite barrier for electrons. Figure 6(b) shows with more detail $2 J(R)$ for $L \geqslant 1$ and
3D. For $L \leqslant 1$ [see Fig. 6(a)], the exchange energy at very small $R<1$ shows a maximum for 2D and a minimum for $3 \mathrm{D}$ with intermediate values for QWs of different $L$; however, for $R>2$ the situation is inverted and the 3D value is larger than the value obtained in a $\mathrm{QW}$ or in $2 \mathrm{D}$ [see the inset of Fig. 6(a)].

\section{DISCUSSION}

The exchange energy $2 J(R)$ between two electrons bound to donors, placed in the mid-plane of an infinite $\mathrm{QW}$, has been calculated in units of effective hartree $E_{h}{ }^{*}$, see Sec. III; the distance $R$ between the donors, and the width $L$ of the $\mathrm{QW}$, are expressed in units of effective Bohr radius $a_{B}^{*}$ (see Sec. II A). In order to be specific, we present in Figs. 7(a) and 7(b), in common units of energy and of length, the exchange energy $2 J^{\prime}(r)=2 J\left(R=r / a_{B}^{*}\right) \times E_{h}{ }^{*}$ between two donor-bound electrons as a function of the distance between donors, $r=R a_{B}^{*}$, fixing the width of the infinite $\mathrm{QW}$ at $L^{\prime}=$ $10 \mathrm{~nm}$, for some usually studied semiconductor materials. The parameters for these compounds are indicated in Table I.

We remark in Fig. $7(\mathrm{a})$, that the $2 J^{\prime}(0)$ value is larger for materials with a higher effective hartree energy. Indeed, a value close to $100 \mathrm{meV}$ for $\mathrm{ZnO}$ is obtained, which is almost five times larger than the $2 J^{\prime}(0)$ value for GaAs. Figure 7 (b) shows that, for large interdonor distances, $\mathrm{ZnO}$ exhibits also the smallest value of exchange energy, meanwhile GaAs has the largest exchange energy among the materials used for comparison in this figure. We also remark that, for GaAs, the exchange energy of two electrons localized on donors decreases slightly when the interdonor distance increases, meanwhile for $\mathrm{ZnO}$ the decrease is much faster. In general, the exchange energy is exponentially sensitive to the distance between donors, and this sensitivity depends also strongly on the effective hartree energy which fixes the extension of the electron wavefunction for a given semiconductor material.

Talking about physical applications, the lowest limit of distance for lithographic techniques, and therefore the closest available distance for qubits, is of the order of tens of $\mathrm{nm}$. When the exchange interaction is the main coupling mechanism between spin qubits, a relatively strong exchange interaction is needed, but also needed is a long spin relaxation time; Linpeng et al. [36] have measured in $\mathrm{ZnO}$ bulk, with a doping concentration of the order of $10^{17} \mathrm{~cm}^{-3}$ (i.e., $r \sim 15 \mathrm{~nm}$ ), a relaxation time exceeding $100 \mathrm{~ms}$ applying a magnetic field of $2 \mathrm{~T}$, meanwhile Dzhioev et al. [22] have found a spin

TABLE I. Values of the different parameters determining the electron exchange energy and the spin relaxation time near the insulator-metal transition, in usually studied semiconductor materials.

\begin{tabular}{|c|c|c|c|c|c|c|}
\hline Material & $\begin{array}{l}\text { Effective mass } \\
\qquad m^{*} / m_{0}\end{array}$ & $\begin{array}{c}\text { Dielectric constant } \\
\epsilon_{r}\end{array}$ & $\begin{array}{c}\text { Bohr radius } \\
a_{B}^{*}(\mathrm{~nm})\end{array}$ & $\begin{array}{c}\text { Effective hartree } \\
E_{h}^{*}(\mathrm{meV})\end{array}$ & $\begin{array}{c}\text { Energy band gap } \\
E_{g}(\mathrm{eV})\end{array}$ & $\begin{array}{c}\text { Spin-orbit splitting } \\
\Delta_{S O}(\mathrm{eV})\end{array}$ \\
\hline $\mathrm{ZnO}$ & $0.24^{\mathrm{a}}$ & $7.77^{\mathrm{b}}$ & 1.71 & 108 & $3.44^{\mathrm{c}}$ & $0.0035^{\mathrm{c}}$ \\
\hline $\mathrm{GaN}$ & $0.13^{\mathrm{f}}$ & $9.7^{\mathrm{f}}$ & 3.95 & 37.6 & $3.28^{\mathrm{f}}$ & $0.02^{\mathrm{f}}$ \\
\hline $\mathrm{CdSe}$ & $0.11^{\mathrm{b}}$ & $10.16^{\mathrm{d}}$ & 4.89 & 29.0 & $1.74^{\mathrm{g}}$ & $0.462^{\mathrm{h}}$ \\
\hline $\mathrm{CdTe}$ & $0.09^{\mathrm{i}}$ & $10.31^{\mathrm{j}}$ & 6.1 & 23 & $1.606^{\mathrm{e}}$ & $0.949^{\mathrm{e}}$ \\
\hline
\end{tabular}

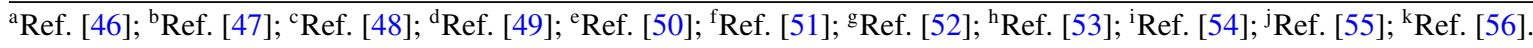


relaxation time of about $100 \mathrm{~ns}$ in GaAs for an interdonor distance around $30 \mathrm{~nm}$. Then, when the coupling between two spin qubits is governed by exchange interaction, the choice of the most suitable material is the result of a compromise between large spin relaxation time and large enough exchange interaction.

In Refs. [22-24], it has been shown that the exchange interaction plays an essential role for the spin relaxation of donorbound electrons near the metal-insulator transition, in which the anisotropic part of the exchange Hamiltonian becomes dominant. Until now, we have only dealt with the isotropic part, but $J$ also appears as a coefficient of the anisotropic terms. The complete exchange Hamiltonian in semiconductor nanostructures is given by [34]

$$
\begin{aligned}
\hat{H}_{\mathrm{exc}}= & 2 J\left[\vec{S}_{1} \cdot \vec{S}_{2} \cos \gamma+\left(\vec{d} \cdot \vec{S}_{1}\right)\left(\vec{d} \cdot \vec{S}_{2}\right)(1-\cos \gamma)\right. \\
& \left.+\vec{d} \cdot\left(\vec{S}_{1} \times \vec{S}_{2}\right) \sin \gamma\right],
\end{aligned}
$$

where $\vec{d}$ is a unit vector and $\gamma$ is a constant proportional to the distance between the two electrons; $\vec{d}$ and $\gamma$ both depend on the crystallographic structure. The first anisotropic term in Eq. (16) corresponds to the pseudodipole interaction, and the second one to the Dzyaloshinskii-Moriya (DM) interaction. The magnitude of $\gamma$ characterizes the strength of the anisotropic part; for small $\gamma$, the anisotropic part is dominated by the DM term.

The dopant concentration at which the insulator-metal transition (Mott transition) appears in 3D is fixed by the expression $n_{\mathrm{Mott}}^{1 / 3} a_{B}^{*}=0.25$ [37], which corresponds to the distance $\approx 3 a_{B}^{*}$ between the donors. The spin relaxation time for localized electrons near the insulator-metal transition, in the insulating phase, is fixed by the anisotropic part of the exchange Hamiltonian. The expression for the spin relaxation time in a QW of thickness $L^{\prime}$ is then given by [22]

$$
\tau_{s a}=\frac{3}{2} \frac{\tau_{c}}{\gamma^{2}}
$$

with $\tau_{\mathrm{c}}$ the characteristic residence time of an electron on a donor:

$$
\tau_{c} \approx \frac{\hbar}{\xi J^{\prime}(r)},
$$

in which $\xi$ is a numerical factor of the order of one, approximately equal to 0.8 in the $3 \mathrm{D}$ case [22]. In general, for a $\mathrm{QW}, \gamma$ can be written for both zinc-blende (ZB) and wurzite structures as

$$
\gamma=2 \pi^{2} \frac{\gamma_{e} b}{E_{h}^{*} a_{B}^{* 3}}\left(\frac{a_{B}^{*}}{L^{\prime}}\right)^{2} R
$$

with $R=r / a_{B}^{*}, b$ a parameter equal to 1 or 4 for $\mathrm{ZB}$ or wurtzite crystals, respectively, and $\gamma_{e}$ is the splitting coefficient related to the Dresselhaus term of the spin-orbit Hamiltonian [38,39]. In the ZB structure, this coefficient $\gamma_{e}^{\mathrm{ZB}}$ is proportional to the spin-orbit constant $\alpha$ (defined in Ref. [40]):

$$
\gamma_{e}^{\mathrm{ZB}}=\frac{\alpha \hbar^{3}}{m^{*} \sqrt{2 m^{*} E_{g}}} .
$$

The substitution of Eq. (20) in Eq. (19) leads to the expression proposed in Ref. [34]. For a wurzite crystal, the

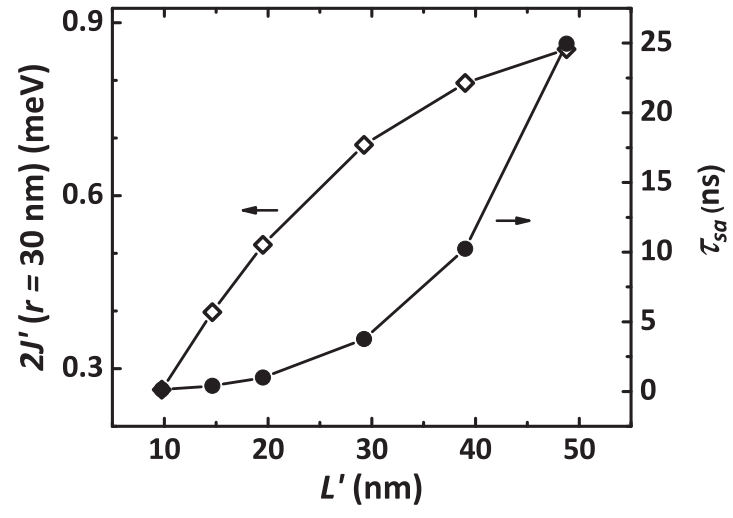

FIG. 8. Electron exchange energy in a common unit of energy (empty diamonds, left axis) and spin relaxation time (full disks, right axis) due to the anisotropic exchange mechanism, for different GaAs QW thicknesses. The distance $r=30 \mathrm{~nm}$ is fixed between two donor-bound electrons.

expression for $\gamma_{e}^{W}$ and values for specific materials can be found in Refs. [38,41].

For GaAs, one of the most studied materials, the interdonor distance for which the Mott transition appears is around $r_{\text {Mott }} \approx 3 a_{B}^{*}=30 \mathrm{~nm}$. In Fig. 8 we show for this material, with $\alpha=0.073$ [40] and $r=30 \mathrm{~nm}$, the calculated electron exchange energy $2 J^{\prime}$ and the spin relaxation time $\tau_{s a}$ as a function of the QW thickness. We remark that $2 J^{\prime}(30 \mathrm{~nm})$ increases as $L^{\prime}$ increases, and that for $L^{\prime} \geqslant 30 \mathrm{~nm}$ the exchange energy starts to go to the $3 \mathrm{D}$ limit value, meanwhile $\tau_{s a}$ increases due to the increase of $L^{\prime}$. The upper limit for $\tau_{s a}$ is fixed by the 3D case, which we have estimated around $130 \mathrm{~ns}$; as already mentioned, the experimental value measured by Dzhioev et al. [22] is around $100 \mathrm{~ns}$.

Spin relaxation properties of $\mathrm{GaN}$ and $\mathrm{ZnO}$ are less known than those of GaAs. These materials seem very interesting because they show very long spin relaxation and decoherence times. Indeed, Beschoten et al. have measured at $5 \mathrm{~K}$ and around $220 \mathrm{mT}$, a spin coherence time of $7 \mathrm{~ns}$ for a doping concentration of $3.5 \times 10^{16} \mathrm{~cm}^{-3}$ in GaN [42].

In a $n$-doped $\mathrm{ZnO}$ epilayer with concentrations close to the metallic zone, S. Ghosh et al. found a spin coherence time of $2 \mathrm{~ns}$ at $\mathrm{T}=30 \mathrm{~K}$ [43]. We now compare this value with the one which can be estimated using the calculated $J$ for a QW. In $\mathrm{ZnO}, \gamma_{e}^{W}$ has been calculated to be $0.33 \mathrm{eV} . \AA^{3}$ (see Refs. [38,41]), two orders of magnitude smaller than for GaAs. By using the parameters given in Table I, and for a doping concentration in the insulating regime near the Mott transition $(r=5 \mathrm{~nm})$, we are then able to estimate a relaxation time $\tau_{s a}^{\mathrm{ZnO}} \approx 16 \mathrm{~ns}$ for a $\mathrm{QW}$ with a thickness equal to $10 \mathrm{~nm}$. This value is larger than the one obtained for a GaAs QW of the same thickness, $\tau_{s a}^{\mathrm{GaAs}} \approx 150$ ps (see Fig. 8), and also larger than the relaxation times measured by Ghosh et al. [43] on $\mathrm{ZnO}$ epilayers close to the Mott transition $(0.5-2 \mathrm{~ns}$, for $\left.n a_{B}^{* 3}=0.01-0.1\right)$. Nevertheless, the cited experimental values may also depend on spin relaxation processes not discussed in this work, and induced by the optical excitation conditions [24]. 
TABLE II. Wavefunctions and energies for the $1 s$ and $2 s$ hydrogenic states in $3 \mathrm{D}$ and $2 \mathrm{D}$.

\begin{tabular}{|c|c|c|c|}
\hline & & Wavefunction $\phi$ & Energy E \\
\hline \multirow[t]{2}{*}{ 3D } & $1 s$ & $\frac{1}{\sqrt{\pi}} Z^{3 / 2} \exp (-Z r)$ & $-Z^{2} / 2$ \\
\hline & $2 s$ & $\frac{1}{2 \sqrt{2 \pi}} Z^{3 / 2} \exp (-Z r / 2)[1-Z r / 2]$ & $-Z^{2} / 8$ \\
\hline \multirow[t]{2}{*}{ 2D } & $1 s$ & $\frac{4}{\sqrt{2 \pi}} Z \exp (-2 Z \rho)$ & $-2 Z^{2}$ \\
\hline & $2 s$ & $\frac{4}{3 \sqrt{6 \pi}} Z \exp (-2 Z \rho / 3)[1-4 Z \rho / 3]$ & $-2 Z^{2} / 9$ \\
\hline
\end{tabular}

\section{CONCLUSION}

We obtained a general expression for the exchange energy of two electrons bound to donors placed in the middle of an infinite QW, valid for any interdonor distance and for any QW thickness. This tool allows one, in particular, to calculate the spin relaxation time near the insulator-metal transition in QWs made of widely used II-VI and III-V direct-band-gap materials. Due to the low values of the electron exchange energy and the spin-orbit interaction in $\mathrm{ZnO}$, we deduce that the spin relaxation time in $\mathrm{ZnO}$ near the metal-insulator transition should be larger than the one found in GaAs, and then $\mathrm{ZnO}$ could be a suitable material for quantum information, provided the entanglement mechanism between spin qubits be different from the electron exchange interaction.

\section{APPENDIX A: $1 s$ AND $2 s$ HYDROGENIC STATES CENTERED IN AN INFINITE QUANTUM WELL}

\section{Method}

A positive electric charge $Z e$ ( $e$ : elementary charge) is at the origin of Cartesian coordinates $x y z$. The Hamiltonian $H$ of an electron (charge $-e$ ) in the Coulomb potential of $Z e$ is

$$
H=-\frac{\Delta}{2}-\frac{Z}{r},
$$

where $\Delta$ is the Laplacian operator and $r$ is the distance of the electron to the origin. The distances are expressed in units of effective (bulk) Bohr radius $a_{B}^{*}$, and energies in units of effective hartree $E_{h}{ }^{*}$ (as mentioned in the beginning of Sec. II A). The $1 s$ and $2 s$ states in bulk $\left(r=\sqrt{x^{2}+y^{2}+z^{2}}\right)$ and in $2 \mathrm{D}\left(\rho=\sqrt{x^{2}+y^{2}}\right)$ are shown in Table II.

We seek the $1 s$ and $2 s$ states when the charge $Z e$ is placed in the middle of a $\mathrm{QW}$ of thickness $L$, simply modeled by two infinite barriers located at $z= \pm L / 2$; the confinement potential is supposed to be zero inside the QW $(|z|<L / 2)$. The $1 s$ and $2 s$ wavefunctions centered within this QW are taken in the following forms:

$$
\begin{aligned}
& \phi_{1}(\rho, z)=A_{1} \exp \left(-\alpha_{1} r\right) \cos \left(\pi \frac{z}{L}\right) \\
& \phi_{2}(\rho, z)=A_{2} \exp \left(-\alpha_{2} r\right)\left[1-\alpha_{3} r\right] \cos \left(\pi \frac{z}{L}\right),
\end{aligned}
$$

where $r=\sqrt{\rho^{2}+z^{2}}$. The postulated $\cos (\pi z / L)$ envelope function is a single sinusoid arch, and ensures that the wavefunctions $\phi_{j}(\rho, z), j=1$ or 2 , vanish at the boundaries of the QW. The dependences on $r$ of both $\phi_{j}$ respect the ones which are found in the 2D $(L \rightarrow 0)$ and 3D $(L \rightarrow \infty)$ limits. In the following of this Appendix, the prefactors $A_{j}$ are fixed by normalization; $\phi_{1}(\rho, z)$ and its energy $E_{1}$ are first determined by means of the variational method; afterwards, using the $2 s-1 s$ orthogonality and the variational method, $\phi_{2}(\rho, z)$ and its energy $E_{2}$ are obtained.

\section{1s state}

The normalization condition of the $\phi_{1}(\rho, z)$ wavefunction gives

$$
A_{1}^{2}=\frac{\alpha_{1}^{3}}{\pi N\left(\alpha_{1} L\right)}
$$

with

$$
\begin{aligned}
N(X)= & 1-\frac{\pi^{2}}{X^{2}+\pi^{2}}+\frac{\pi^{4} / 2}{\left[X^{2}+\pi^{2}\right]^{2}} \\
& -\frac{\pi^{2}}{4}\left(\frac{X+4}{X^{2}+\pi^{2}}-\frac{2 \pi^{2}}{\left[X^{2}+\pi^{2}\right]^{2}}\right) e^{-X} .
\end{aligned}
$$

The forms of the normalization factors of the 3D and 2D $1 s$ wavefunctions can be retrieved from this expression: $A_{1} \rightarrow$ $\alpha_{1}^{3 / 2} / \sqrt{\pi}$ for $L \rightarrow \infty$, and $A_{1} \sqrt{L / 2} \rightarrow \alpha_{1} \sqrt{2 / \pi}$ for $L \rightarrow 0$.

The parameter $\alpha_{1}$ is obtained by minimization of the mean energy $\tilde{E}_{1}=\left\langle\phi_{1}|H| \phi_{1}\right\rangle$, which is the sum of a kinetic term (possessing, after calculation, a remarkably simple form):

$$
\left\langle\phi_{1}\left|-\frac{\Delta}{2}\right| \phi_{1}\right\rangle=\frac{\alpha_{1}^{2}}{2}+\frac{\pi^{2}}{2 L^{2}},
$$

and a potential term due to the Coulomb interaction:

$$
\left\langle\phi_{1}\left|-\frac{Z}{r}\right| \phi_{1}\right\rangle=-Z \alpha_{1} \frac{C\left(\alpha_{1} L\right)}{N\left(\alpha_{1} L\right)},
$$

with

$$
C(X)=1-\frac{1}{2} \frac{\pi^{2}}{X^{2}+\pi^{2}}\left(1+e^{-X}\right)
$$

Defining $g(X)=X C(X) / N(X)$, which is a smooth function close to the identity $[g(X) \approx X]$, the minimum of $\tilde{E}_{1}$ is found to be reached when $\alpha_{1}$ is solution of the equation

$$
\frac{\alpha_{1} L}{g^{\prime}\left(\alpha_{1} L\right)}=Z L
$$

After a numerical determination of the derivative $g^{\prime}(X)$ of the function $g(X)$, the left-hand side of the above equation can be calculated for a value of the parameter $\alpha_{1} L$. One then obtains the thickness $L$ for which the starting parameter $\alpha_{1} L$ corresponds to the minimum of $\tilde{E}_{1}$; finally, the associated $\alpha_{1}$ value is calculated through $\alpha_{1}=\left(\alpha_{1} L\right) / L$, and the energy $E_{1}$ of $\phi_{1}$ by substitution of $\alpha_{1}$ in the expression for $\tilde{E}_{1}$. The procedure can be repeated for any starting parameter $\alpha_{1} L$, and then allows one to get $\alpha_{1}$ and $E_{1}$, and also $A_{1}$, as a function of the thickness $L$ of the QW, as shown in Fig. 9 for $Z=1$ and $Z=2$. One can remark that parameter $\alpha_{1}(Z=1)$ for thickness $L$ coincides with $\alpha_{1}(Z) / Z$ for thickness $Z L$. The prefactor $A_{1}$ goes to $\sqrt{Z^{3} / \pi}$ (3D limit) for $L \gg 1$, and behaves as $4 Z / \sqrt{\pi L}$ for very small $L$ [see Figs. 9(a) and 

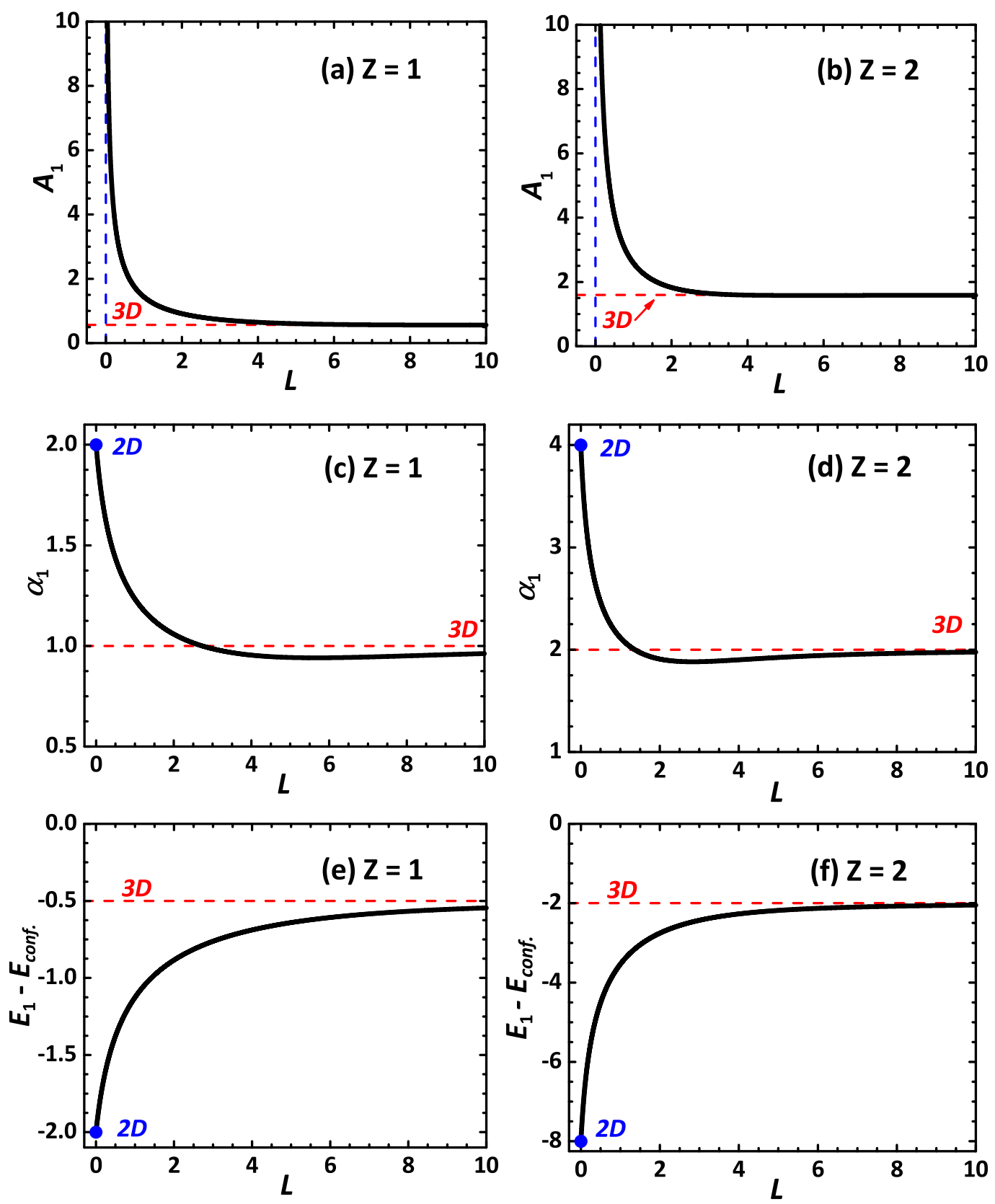

FIG. 9. Parameters $A_{1}$ and $\alpha_{1}$ of the $\phi_{1}$ wavefunction, and its energy $E_{1}$, as a function of the thickness $L$ of the QW (thick continuous curves); the thin red dashed lines correspond to the 3D case. (a), (b): $A_{1}$ vs $L$ for $Z=1, Z=2$. (c), (d): $\alpha_{1}$ vs $L$ for $Z=1, Z=2$. (e), (f): $E_{1}-E_{\text {conf }}$ vs $L$ for $Z=1, Z=2$, where $E_{\text {conf }}=\pi^{2} / 2 L^{2}$.

9(b)]. The parameter $\alpha_{1}$ goes to $Z$ (3D limit) for $L \gg 1$, and to $2 Z$ (2D limit) for vanishing $L$ [see Figs. 9(c) and 9(d)]. For decreasing values of $L$, the extension of the $\phi_{1}$ wavefunction first inflates slightly in the $x y$ plane $\left(\alpha_{1}\right.$ becoming a little smaller than $Z$ ), and secondly globally shrinks for smaller values of $L$. The energy $E_{1}$, when considered by reference to the confinement energy $E_{\text {conf }}=\pi^{2} / 2 L^{2}$, increases monotonically with $L$ from $-2 Z^{2}$ (2D value) to $-Z^{2} / 2$ (3D value) [see Figs. 9(e) and 9(f)].

\section{3. $2 s$ state}

The orthogonality condition between the $\phi_{1}(\rho, z)$ and $\phi_{2}(\rho, z)$ wavefunctions implies the following relationship be- tween $\alpha_{1}, \alpha_{2}$, and $\alpha_{3}$ :

$$
\alpha_{3}=\beta \frac{N(\beta L)}{N(\beta L)+M(\beta L)},
$$

with $\beta=\frac{\alpha_{1}+\alpha_{2}}{2}$ and

$$
\begin{aligned}
M(X)= & \frac{1}{2}-\frac{3 \pi^{2} / 2}{X^{2}+\pi^{2}}+\frac{9 \pi^{4} / 4}{\left[X^{2}+\pi^{2}\right]^{2}}-\frac{\pi^{6}}{\left[X^{2}+\pi^{2}\right]^{3}} \\
& -\frac{\pi^{2}}{4}\left(\frac{1}{2}+\frac{3 X+6-\pi^{2} / 2}{X^{2}+\pi^{2}}-\pi^{2} \frac{2 X+9}{\left[X^{2}+\pi^{2}\right]^{2}}\right. \\
& \left.+\frac{4 \pi^{4}}{\left[X^{2}+\pi^{2}\right]^{3}}\right) e^{-X} .
\end{aligned}
$$


The relationships between $\alpha_{1}, \alpha_{2}$ and $\alpha_{3}$ in 3D and in 2D can be retrieved as limiting cases of the above expressions: $\alpha_{3} \rightarrow$ $\left(\alpha_{1}+\alpha_{2}\right) / 3$ when $L \rightarrow \infty$ and $\alpha_{3} \rightarrow\left(\alpha_{1}+\alpha_{2}\right) / 2$ when $L \rightarrow 0$.

The normalization condition of the $\phi_{2}(\rho, z)$ wavefunction gives

$$
\begin{aligned}
A_{2}^{2}= & \frac{\alpha_{2}^{3}}{\pi}\left\{N\left(\alpha_{2} L\right)\left[1-2 \frac{\alpha_{3}}{\alpha_{2}}+\frac{3}{2} \frac{\alpha_{3}^{2}}{\alpha_{2}^{2}}\right]\right. \\
& \left.+M\left(\alpha_{2} L\right)\left[-2 \frac{\alpha_{3}}{\alpha_{2}}+\frac{3}{2} \frac{\alpha_{3}^{2}}{\alpha_{2}^{2}}\right]+\frac{1}{8} Q\left(\alpha_{2} L\right) \frac{\alpha_{3}^{2}}{\alpha_{2}^{2}}\right\}^{-1},
\end{aligned}
$$

with

$$
\begin{aligned}
Q(X)= & 3+3 X^{4} \frac{X^{4}-6 \pi^{2} X^{2}+\pi^{4}}{\left[X^{2}+\pi^{2}\right]^{4}}-\frac{\pi^{2}}{2}\left(\frac{X^{3}}{X^{2}+\pi^{2}}\right. \\
& +3 X^{2} \frac{3 X^{2}+\pi^{2}}{\left[X^{2}+\pi^{2}\right]^{2}}+6 X \frac{6 X^{4}+3 \pi^{2} X^{2}+\pi^{4}}{\left[X^{2}+\pi^{2}\right]^{3}} \\
& \left.+6 \frac{10 X^{6}+5 \pi^{2} X^{4}+4 \pi^{4} X^{2}+\pi^{6}}{\left[X^{2}+\pi^{2}\right]^{4}}\right) e^{-X} .
\end{aligned}
$$

The energy $E_{2}$ is obtained as the minimum of the mean energy $\tilde{E}_{2}=\left\langle\phi_{2}|H| \phi_{2}\right\rangle$, which only depends on $\alpha_{2}\left(\alpha_{1}\right.$ is known, $\alpha_{3}$ is a function of $\alpha_{1}$ and $\left.\alpha_{2}\right) . \tilde{E}_{2}$ is the sum of a kinetic term:

$$
\begin{aligned}
\left\langle\phi_{2}\left|-\frac{\Delta}{2}\right| \phi_{2}\right\rangle= & \frac{1}{4}\left(\frac{\pi A_{2}^{2}}{\alpha_{2}^{3}}\right)\left\{\frac { \pi ^ { 2 } } { L ^ { 2 } } \left[Q_{1}\left(\alpha_{2} L\right)-\frac{\alpha_{3}}{\alpha_{2}} Q_{2}\left(\alpha_{2} L\right)\right.\right. \\
& \left.+\frac{\alpha_{3}^{2}}{4 \alpha_{2}^{2}} Q_{3}\left(\alpha_{2} L\right)\right]+2 \alpha_{2}^{2} C\left(\alpha_{2} L\right) \\
& +\alpha_{3}^{2}\left[N\left(\alpha_{2} L\right)+2 M\left(\alpha_{2} L\right)\right] \\
& \left.+2 \alpha_{2} \alpha_{3}\left[C\left(\alpha_{2} L\right)-2 N\left(\alpha_{2} L\right)\right]\right\}, \quad \text { (A9a) }
\end{aligned}
$$

with

$$
\begin{aligned}
& Q_{1}(X)=1-\left(1+\frac{X}{2}\right) e^{-X} \\
& Q_{2}(X)=3-\left(3+2 X+\frac{1}{2} X^{2}\right) e^{-X} \\
& Q_{3}(X)=12-\left(12+9 X+3 X^{2}+\frac{1}{2} X^{3}\right) e^{-X},
\end{aligned}
$$

and a potential term due to the Coulomb interaction:

$$
\begin{aligned}
\left\langle\phi_{2}\left|-\frac{Z}{r}\right| \phi_{2}\right\rangle= & -Z \frac{\pi A_{2}^{2}}{\alpha_{2}^{2}}\left\{C\left(\alpha_{2} L\right)+N\left(\alpha_{2} L\right)\right. \\
& \left.\times\left[-2 \frac{\alpha_{3}}{\alpha_{2}}+\frac{\alpha_{3}^{2}}{\alpha_{2}^{2}}\right]+M\left(\alpha_{2} L\right) \frac{\alpha_{3}^{2}}{\alpha_{2}^{2}}\right\} .
\end{aligned}
$$

Finding the parameter $\alpha_{2}$ [and consequently $\alpha_{3}$, with Eq. (A7a)] which minimizes $\tilde{E}_{2}$ (at given $L$ ) requires a long numerical procedure, which is in contrast with the much easier work needed for the minimization of $\tilde{E}_{1}$. Figure 10 shows $A_{2}$, $\alpha_{2}, \alpha_{3}$ and $E_{2}$ concerning the $\phi_{2}$ wavefunction, for different values of the thickness $L$, with $Z=1$ and $Z=2: A_{2}$ and $\alpha_{2}$ behave with $L$ just as $A_{1}$ and $\alpha_{1}$ do; $\alpha_{3}$ monotonically decreases with $L$; however, $E_{2}$, considered by reference to the confinement energy $E_{\mathrm{conf}}=\pi^{2} / 2 L^{2}$, goes with $L$ from $-2 Z^{2} / 9$ (2D value) to $-Z^{2} / 8$ (3D value) but nonmonotonically [see Figs. 10(e) and 10(f)].

\section{APPENDIX B: EXCHANGE ENERGY OF A "HELIUM ATOM” CENTERED IN AN INFINITE QUANTUM WELL}

\section{Exact results in 3D}

The nonrelativistic energies of the (singlet) $(1 s)^{2}$ ground state and of the first-excited triplet $1 s 2 s$ state of the helium atom in 3D are known with an excellent precision: in Ref. [44] they are calculated with 20 significant digits. We only write here the first of them:

$$
E_{S}^{\text {exact }}=-2.9037, \quad E_{A}^{\text {exact }}=-2.1752,
$$

in units of effective hartree (see the beginning of Sec. II A). The subscript $S$, or $A$, recalls that the orbital part of the state is symmetric, or antisymmetric, under permutation of both electrons of the atom. The exchange energy $2 J_{0}=E_{A}-E_{S}$ is then exactly, in 3D:

$$
2 J_{0}^{\text {exact }}=0.7285 \text {. }
$$

\section{Exact results in $2 D$}

In $2 \mathrm{D}$, the nonrelativistic energies of the $(1 s)^{2}$ ground state and the first-excited triplet $1 s 2 s$ state of the helium atom are also known with a very good accuracy: in Ref. [45] they are calculated with 13 significant digits. We write here the first of them:

$$
E_{S}^{\text {exact }}=-11.900, \quad E_{A}^{\text {exact }}=-8.296,
$$

in units of effective hartree. The exchange energy is then exactly, in 2D:

$$
2 J_{0}^{\text {exact }}=3.604
$$

\section{Calculation of $2 J_{0}$ in an infinite quantum well}

The Hamiltonian describing both electrons in a helium atom is written as

$$
\hat{H}=-\frac{\Delta_{1}}{2}-\frac{\Delta_{2}}{2}-\frac{Z}{r_{1}}-\frac{Z}{r_{2}}+\frac{1}{r_{12}},
$$

where $\Delta_{j}$ is the Laplacian operator acting on electron $j$ ( $j=$ 1 or 2) and $r_{j}$ is the distance of electron $j$ to the origin of the coordinates, where the $Z=2$ nucleus is located; $r_{12}=$ $\left|\vec{r}_{2}-\vec{r}_{1}\right|$ is the distance between the electrons.

The ground energy $E_{S}$ of the "helium atom" centered in a QW with infinite barriers is determined here by the variational method. The ground-state wavefunction $\Psi_{S}\left(\vec{r}_{1}, \vec{r}_{2}\right)$ of the pair of electrons 1 and 2 is taken as the product of two one-electron 1s wavefunctions: $\Psi_{S}\left(\vec{r}_{1}, \vec{r}_{2}\right)=\phi_{1}\left(\vec{r}_{1}\right) \phi_{1}\left(\vec{r}_{2}\right)$; the mean energy $\tilde{E}_{S}=\left\langle\Psi_{S}|\hat{H}| \Psi_{S}\right\rangle$ is studied as a function of the 

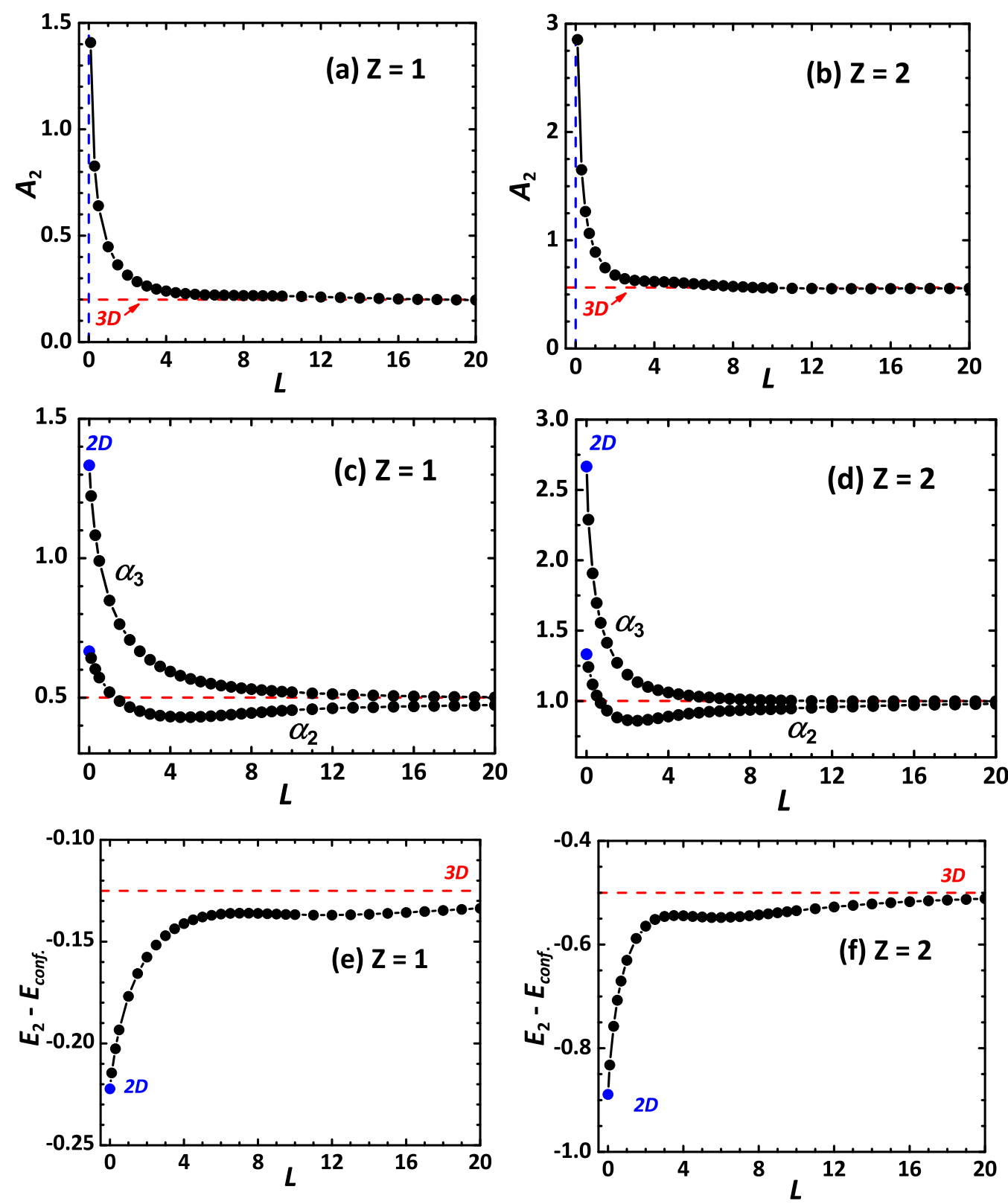

FIG. 10. Parameters $A_{2}, \alpha_{2}$ and $\alpha_{3}$ of the $\phi_{2}$ wavefunction, and its energy $E_{2}$, as a function of the thickness $L$ of the QW (circular dots; continuous curves: guides for the eyes); the thin red dashed lines correspond to the 3D case. (a), (b): $A_{2}$ vs $L$ for $Z=1, Z=2$. (c), (d): $\alpha_{2}$ and $\alpha_{3}$ vs $L$ for $Z=1, Z=2$. (e), (f): $E_{2}-E_{\text {conf }}$ vs $L$ for $Z=1, Z=2\left(E_{\text {conf }}=\pi^{2} / 2 L^{2}\right)$.

parameter $\alpha_{1}$, which now can vary (see Appendix A 1 for the definitions of $\phi_{1}$ and $\alpha_{1}$ ), and its minimum is taken for the value of $E_{S} . \tilde{E}_{S}$ is the sum of a kinetic term:

$$
\left\langle\Psi_{S}\left|-\frac{\Delta_{1}}{2}-\frac{\Delta_{2}}{2}\right| \Psi_{S}\right\rangle=\alpha_{1}^{2}+\frac{\pi^{2}}{L^{2}},
$$

a potential term due to the Coulomb interaction of the electrons with the $Z=2$ nucleus:

$$
\left\langle\Psi_{S}\left|-\frac{2}{r_{1}}-\frac{2}{r_{2}}\right| \Psi_{S}\right\rangle=-4 \alpha_{1} \frac{C\left(\alpha_{1} L\right)}{N\left(\alpha_{1} L\right)}
$$

$[N(X)$ and $C(X)$ : see Appendix A], and a Coulomb electron-electron term:

$$
\left\langle\Psi_{S}\left|\frac{1}{r_{12}}\right| \Psi_{S}\right\rangle=32 \pi \int_{0}^{\infty} \int_{0}^{\infty} \frac{\rho_{q} d \rho_{q} d z_{q}}{\rho_{q}^{2}+z_{q}^{2}}\left[\int_{0}^{\frac{L}{2}} d z \cos \left(z_{q} z\right) \int_{0}^{\infty} d \rho \rho J_{0}\left(\rho_{q} \rho\right) \phi_{1}(\rho, z)^{2}\right]^{2},
$$

expressed using the fact that $1 / r$ is the inverse Fourier transform of $4 \pi / q^{2}\left[J_{0}(x)\right.$ is the Bessel function of first kind and of zeroth order]. This integral is calculated numerically. 
The first-excited energy $E_{A}$ of the "helium atom" centred in a QW with infinite barriers is determined perturbatively here: the electron-electron interaction $1 / r_{12}$ is treated as a small correction to the state $\Psi_{A}\left(\vec{r}_{1}, \vec{r}_{2}\right)=\left[\phi_{1}\left(\vec{r}_{1}\right) \phi_{2}\left(\vec{r}_{2}\right)-\phi_{2}\left(\vec{r}_{1}\right) \phi_{1}\left(\vec{r}_{2}\right)\right] / \sqrt{2}$ of energy $E_{1}+E_{2}\left(\phi_{1}, \phi_{2}, E_{1}\right.$, and $E_{2}$ are given in Appendix A). The energy $E_{A}$ is of the form $E_{A}=E_{1}+E_{2}+\Delta E$, the corrective term being $\Delta E=\left\langle\Psi_{A}\left|1 / r_{12}\right| \Psi_{A}\right\rangle ; \Delta E$ is the sum of a direct term:

$$
\begin{aligned}
\iint d^{3} r_{1} d^{3} r_{2} \frac{\phi_{1}\left(\vec{r}_{1}\right)^{2} \phi_{2}\left(\vec{r}_{2}\right)^{2}}{r_{12}}= & 32 \pi \int_{0}^{\infty} \int_{0}^{\infty} \frac{\rho_{q} d \rho_{q} d z_{q}}{\rho_{q}^{2}+z_{q}^{2}}\left[\int_{0}^{\frac{L}{2}} d z \cos \left(z_{q} z\right) \int_{0}^{\infty} d \rho \rho J_{0}\left(\rho_{q} \rho\right) \phi_{1}(\vec{r})^{2}\right] \\
& \times\left[\int_{0}^{\frac{L}{2}} d z \cos \left(z_{q} z\right) \int_{0}^{\infty} d \rho \rho J_{0}\left(\rho_{q} \rho\right) \phi_{2}(\vec{r})^{2}\right],
\end{aligned}
$$

and an exchange term:

$$
\iint d^{3} r_{1} d^{3} r_{2} \frac{\phi_{1}\left(\vec{r}_{1}\right) \phi_{2}\left(\vec{r}_{2}\right) \phi_{2}\left(\vec{r}_{1}\right) \phi_{1}\left(\vec{r}_{2}\right)}{r_{12}}=32 \pi \int_{0}^{\infty} \int_{0}^{\infty} \frac{\rho_{q} d \rho_{q} d z_{q}}{\rho_{q}^{2}+z_{q}^{2}}\left[\int_{0}^{\frac{L}{2}} d z \cos \left(z_{q} z\right) \int_{0}^{\infty} d \rho \rho J_{0}\left(\rho_{q} \rho\right) \phi_{1}(\vec{r}) \phi_{2}(\vec{r})\right]^{2} .
$$

In $3 \mathrm{D}$ and in $2 \mathrm{D}$, calculating $E_{S}$ by the variational method and $E_{A}$ perturbatively gives satisfactory values (for our purpose) of the exchange energy $2 J_{0}=E_{A}-E_{S}$ : we so obtain $2 J_{0}=-2.124+2.848=0.724$ in $3 \mathrm{D}$, and $-7.948+$ $11.635=3.687$ in $2 \mathrm{D}$, which are $0.6 \%$ smaller, and $2 \%$ larger, than the exact values, respectively. We are then confident that our calculated exchange energies $2 J_{0}$ of a "helium atom" centred in infinite QWs of different thicknesses, possess an uncertainty of the $1 \%$ order.

Figure 11 shows our calculated values of the ground energy $E_{S}$ and first-excited energy $E_{A}$, as a function of the thickness $L$ of the QW; the exact values in 2D $(L \rightarrow 0)$ and in 3D $(L \rightarrow$ $\infty)$ are also indicated. $E_{S}$ and $E_{A}$ monotonically increase with the thickness $L$, and their difference, which is $2 J_{0}$, monotonically decreases, as shown in Fig. 2.

\section{APPENDIX C: NEW INTERPOLATED FORMULAS FOR THE 3D AND 2D EXCHANGE ENERGIES}

Section III A described the procedure of Ref. [32] to build an interpolated expression of the exchange energy $2 J(R)$ in

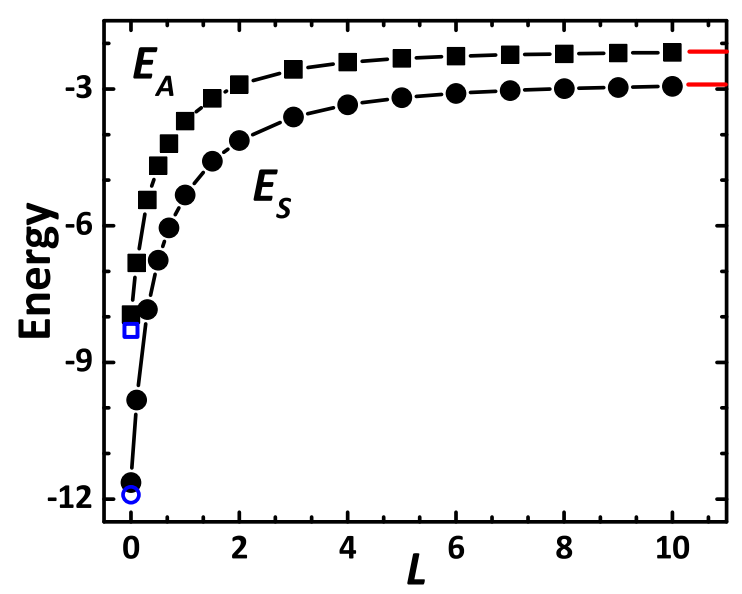

FIG. 11. Ground energy $E_{S}$ (full disks) and first-excited energy $E_{A}$ (full squares) of a "helium atom", as a function of the thickness $L$ of the infinite QW; the continuous curves are guides for the eyes. The empty circle and empty square, at $L=0$, are the exact values in $2 \mathrm{D}$. The exact values in $3 \mathrm{D}$ are indicated by two horizontal red segments, on the right of the graph.
3D and 2D. In Eq. (14), in which $A$ is the only remaining free parameter, it is possible, alternatively, to impose that, for $R \gg 1$, the interpolated $2 J(R)$ be in coincidence with the standard asymptotic form (11). We then obtain the following expression for $A$ :

$$
A=\left(C / 2 J_{0}\right)^{1 / \beta} / \mathrm{e}
$$

So we can propose new interpolated formulas for the exchange energy $2 J(R)$ in $3 \mathrm{D}$ and $2 \mathrm{D}$, using values of $2 J_{0}$ which are, nowadays, known with very good accuracy (see Appendix B):

$$
\begin{aligned}
2 J_{3 \mathrm{D}}(R)= & 0.729\left(1+0.259 R^{2}\right)^{\frac{5}{4}} \exp [-0.003 R \\
& -1.271 R \arctan (0.509 R)] \\
2 J_{2 \mathrm{D}}(R)= & 3.604\left(1+1.549 R^{2}\right)^{\frac{7}{8}} \exp [-0.579 R \\
& -2.178 R \arctan (1.245 R)] .
\end{aligned}
$$

These expressions give $2 J(R)$ values close to the corresponding ones of Ref. [32] for $R$ of the order the order of unity, and are more satisfactory, by construction, for large values of $R$.

\section{APPENDIX D: CORRELATION FUNCTION FOR THE 3D CASE}

In Sec. II B, we have found that, in the case of an infinite QW, the correlation function satisfies

$$
\chi\left(\vec{r}_{1}, \vec{r}_{2}\right)=\chi_{3 \mathrm{D}}\left(\vec{r}_{1}, \vec{r}_{2}\right)^{1 / \alpha_{1}}
$$

where $\chi_{3 \mathrm{D}}\left(\vec{r}_{1}, \vec{r}_{2}\right)$ is given by the expression [30]:

$$
\begin{aligned}
\chi_{3 \mathrm{D}}\left(\vec{r}_{1}, \vec{r}_{2}\right) \\
=\frac{2 a\left(2 a+x_{1}+x_{2}\right)}{\left(a-x_{1}\right)\left(a+x_{2}\right)} \exp \left(-\frac{a+x_{1}}{2 a}\right) \\
\quad \times\left\{\frac{\sqrt{\left(x_{1}-x_{2}\right)^{2}+\rho_{12}^{2}}+x_{2}-x_{1}}{\sqrt{\left(2 a+x_{1}+x_{2}\right)^{2}+\rho_{12}^{2}}+2 a+x_{1}+x_{2}}\right\}^{1 / 2}
\end{aligned}
$$


for $x_{1}+x_{2}<0$, and

$$
\chi_{3 \mathrm{D}}\left(\vec{r}_{1}, \vec{r}_{2}\right)=\frac{2 a\left(2 a-x_{1}-x_{2}\right)}{\left(a-x_{1}\right)\left(a+x_{2}\right)} \exp \left(-\frac{a-x_{2}}{2 a}\right)\left\{\frac{\sqrt{\left(x_{1}-x_{2}\right)^{2}+\rho_{12}^{2}}+x_{2}-x_{1}}{\sqrt{\left(2 a-x_{1}-x_{2}\right)^{2}+\rho_{12}^{2}}+2 a-x_{1}-x_{2}}\right\}^{1 / 2}
$$

for $x_{1}+x_{2}>0$; the notation $\rho_{12}=\sqrt{\left(y_{1}-y_{2}\right)^{2}+\left(z_{1}-z_{2}\right)^{2}}$ is employed.

[1] D. P. DiVincenzo, Science 270, 255 (1995).

[2] M. A. Nielsen and I. L. Chuang, Quantum Computation and Quantum Information (Cambridge University Press, Cambridge, 2000).

[3] J. Clarke and F. K. Wilhelm, Nature (London) 453, 1031 (2008).

[4] M. H. Devoret and R. J. Schoelkopf, Science 339, 1169 (2013).

[5] R. Hanson and D. D. Awschalom, Nature (London) 453, 1043 (2008).

[6] J. J. L. Morton and B. W. Lovet, Annu Rev. Condens. Matter Phys. 2, 189 (2011).

[7] D. Loss and D. P. DiVincenzo, Phys. Rev. A 57, 120 (1998).

[8] G. Buckard, D. Loss, and D. P. DiVincenzo, Phys. Rev. B 59, 2070 (1999).

[9] B. E. Kane, Nature (London) 393, 133 (1998).

[10] P. M. Koenraad and M. E. Flatté, Nat. Mater. 10, 91 (2011).

[11] F. Jelezko and J. Wrachtrup, Phys. Status Solidi A 203, 3207 (2006).

[12] M. W. Doherty, N. B. Manson, P. Delaney, F. Jelezko, J. Wrachtrup, and L. C. L. Hollenberg, Phys. Rep. 528, 1 (2013).

[13] W. F. Koehl, B. B. Buckley, F. J. Heremans, G. Calusine, and D. D. Awschalom, Nature (London) 479, 84 (2011).

[14] J. M. Kikkawa and D. D. Awschalom, Phys. Rev. Lett. 80, 4313 (1998).

[15] R. Vrijen, E. Yablonovitch, K. Wang, H. W. Jiang, A. Balandin, V. Roychowdhury, T. Mor, and D. Di Vincenzo, Phys. Rev. A 62, 012306 (2000).

[16] P. H. Kasai, Phys. Rev. 130, 989 (1963).

[17] H. Horn, A. Balocchi, X. Marie, A. Bakin, A. Waag, M. Oestreich, and J. Hübner, Phys. Rev. B 87, 045312 (2013).

[18] J. Tribollet, E. Aubry, G. Karczewski, B. Sermage, F. Bernardot, C. Testelin, and M. Chamarro, Phys. Rev. B 75, 205304 (2007).

[19] M. Chamarro, F. Bernardot, and C. Testelin, J. Phys: Condens Matter 19, 445007 (2007).

[20] K. De Greve, S. M. Clark, D. Sleiter, K. Sanaka, T. D. Ladd, M. Panfilova, A. Pawlis, K. Lischka, and Y. Yamamoto, Appl. Phys. Lett. 97, 241913 (2010).

[21] Y. M. Kim, D. Sleiter, K. Sanaka, D. Reuter, K. Lischka, Y. Yamamoto, and A. Pawlis, Current Appl. Phys. 14, 1234 (2014).

[22] R. I. Dzhioev, K. V. Kavokin, V. L. Korenev, M. V. Lazarev, B. Ya. Meltser, M. N. Stepanova, B. P. Zakharchenya, D. Gammon, and D. S. Katzer, Phys. Rev. B 66, 245204 (2002).

[23] R. I. Dzhioev, V. L. Korenev, I. A. Merkulov, B. P. Zakharchenya, D. Gammon, Al. L. Efros, and D. S. Katzer, Phys. Rev. Lett. 88, 256801 (2002).

[24] K. V. Kavokin, Semicond. Sci. Technol. 23, 114009 (2008).
[25] A. Imamoglu, D. D. Awschalom, G. Burkard, D. P. DiVincenzo, D. Loss, M. Sherwin, and A. Small, Phys. Rev. Lett. 83, 4204 (1999).

[26] W. Yao, R B. Liu, and L. J. Sham, Phys. Rev. Lett. 95, 030504 (2005).

[27] C. Piermarocchi, P. Chen, L. J. Sham, and D. G. Steel, Phys. Rev. Lett. 89, 167402 (2002).

[28] E. Pazy, E. Biolatti, T. Calarco, I. D'Amico, P. Zanardi, F. Rossi, and P. Zoller, Europhys. Lett. 62, 175 (2003).

[29] D. P. DiVincenzo, D. Bacon, J. Kempe, G. Burkard, K. B. Whaley, Nature (London) 408, 339 (2000).

[30] L. P. Gor'kov and L. P. Pitaevskii, Dokl. Akad. Nauk SSSR 151, 822 (1963) [Sov. Phys. Doklady 8, 788 (1964)].

[31] C. Herring and M. Flicker, Phys. Rev. 134, A362 (1964).

[32] I. V. Ponomarev, V. V. Flambaum, and A. L. Efros, Phys. Rev. B 60, 5485 (1999).

[33] I. V. Ponomarev, V. V. Flambaum, and A. L. Efros, Phys. Rev. B 60, 15848 (1999).

[34] K. V. Kavokin, Phys. Rev. B 69, 075302 (2004).

[35] W. Heitler and F. London, Z. Phys. 44, 455 (1927).

[36] X. Linpeng, M. L. K. Viitaniemi, A. Vishnuradhan, Y. Kozuka, C. Johnson, M. Kawasaki, and K-M C. Fu, arXiv:1802.03483v3 (2018).

[37] N. F. Mott, Metal Insulator Transitions (Taylor and Francis, London, 1990).

[38] J. Y. Fu and M. W. Wu, J. Appl. Phys. 104, 093712 (2008).

[39] N. J. Harmon, W. O. Putikka, and R. Joynt, Phys. Rev. B 79, 115204 (2009).

[40] G. E. Pikus and A. M. Titkov, in Optical Orientation, edited by F. Meier and B. P. Zachachrenya (North-Holland, Amsterdam, 1984).

[41] L. C. Lew Yan Voon, M. Willatzen, M. Cardona, and N. E. Christensen, Phys. Rev. B 53, 10703 (1996).

[42] B. Beschoten, E. Johnston-Halperin, D. K. Young, M. Poggio, J. E. Grimaldi, S. Keller, S. P. DenBaars, U. K. Mishra, E. L. Hu, and D. D. Awschalom, Phys. Rev. B 63, 121202 (2001).

[43] S. Ghosh, V. Sih, W. H. Lau, and D. D. Awschalom, Appl. Phys. Lett. 86, 232507 (2005).

[44] D. T. Aznabayev, A. K. Bekbaev, I. S. Ishmukhamedov, and V. I. Korobov, Phys. Part. Nucl. Lett. 12, 689 (2015).

[45] L. Hilico, B. Grémaud, T. Jonckheere, N. Billy, and D. Delande, Phys. Rev. A 66, 022101 (2002).

[46] M. Oshikiri, Y. Imanaka, F. Aryasetiawan, and G. Kido, Physica B 298, 472 (2001).

[47] N. Ashkenov et al., J. Appl. Phys. 93, 126 (2003).

[48] W. J. Fana, J. B. Xiab, P. A. Agus, S. T. Tan, S. F. Yu, and X. W. Sun, J. Appl. Phys. 99, 013702 (2006). 
[49] R. Geick, C. H. Perry, and S. S. Mitra, J. Appl. Phys. 37, 1994 (1966).

[50] H. Mayer and U. Rössler, Solid State Commun. 87, 81 (1993).

[51] Bougrov et al., in Properties of Advanced Semiconductor Materials GaN, AlN, InN, BN, SiC, SiGe, edited by M. E. Levinshtein, S. L. Rumyantsev, and M.S. Shur (Wiley, New York, 2001).

[52] S. Ninomiya and S. Adachi, J. Appl. Phys. 78, 4681 (1995).
[53] H. Fu, L.W. Wang, and A. Zunger, Phys. Rev. B 59, 5568 (1999).

[54] I. Hernández-Calderón, in II-VI Semiconductor Materials and their Applications, edited by M. C. Tamargo (Taylor and Francis, New York, 2002).

[55] I. Strzalkowski, S. Joshi, and C. R. Crowell, Appl. Phys. Lett. 28, 350 (1976).

[56] W. Nakwaski, Physica B 210, 1 (1995). 